Article

\title{
Chemical Characterization of Craft Filuferru Spirit from Sardinia, Italy
}

\author{
Ilaria Langasco ${ }^{1}\left(\mathbb{D}\right.$, Marco Caredda $^{2}$, Gavino Sanna ${ }^{1, *(\mathbb{C})}$, Angelo Panzanelli ${ }^{1}$, Maria Itria Pilo ${ }^{1}$, \\ Nadia Spano ${ }^{1}$, Giacomo Petretto ${ }^{3}$ and Pietro Paolo Urgeghe ${ }^{3}$ (D) \\ 1 Department of Chemistry and Pharmacy, Sassari University, via Vienna 2, 07100 Sassari, Italy; \\ ilarialangasco@gmail.com (I.L); panza@uniss.it (A.P.); mpilo@uniss.it (M.I.P.); nspano@uniss.it (N.S.) \\ 2 Department for the Reseach on the Animal Production, Agris Sardegna, Loc. Bonassai, S.S. 291, \\ Sassari-Fertilia, Km. 18,600, 07100 Sassari, Italy; caredda.m@gmail.com \\ 3 Department of Agriculture, Sassari University, Viale Italia 39, 07100 Sassari, Italy; gpetretto@uniss.it (G.P.); \\ paolou@uniss.it (P.P.U.) \\ * Correspondence: sanna@uniss.it; Tel.: +39-079-229500; Fax: +39-079-0971-388
}

Received: 15 July 2018; Accepted: 16 August 2018; Published: 20 August 2018

\begin{abstract}
Traditional Filuferru is an ancient spirit from Sardinia, Italy, which is usually obtained from the distillation of wine or grape marc. In this contribution, the results of the first chemical characterization of a wide number of crafts Filuferru samples has been accomplished in terms of an evaluation of the alcoholic strength, qualitative and quantitative gas-chromatography-mass spectrometry (GC-MS) analysis of the volatile composition of the distillate, and its trace element composition by means an inductively coupled plasma-mass spectrometry (ICP-MS) method. Both instrumental methods have been validated and applied on $21 \mathrm{craft}$ samples of Filuferru, whereas one sample of commercial distillate has been analyzed for comparison purposes. Alcoholic strength ranged between 41.0 and $62.4 \%(v / v)$. Sixty volatile compounds were identified and ten of them have been quantified. Analogies and differences with Grappa (i.e., the Italian distilled spirit closer to Filuferru) have been highlighted in the qualitative and quantitative profile of this matrix. Often meaningful amounts of acetaldehyde, ethyl acetate, dietyl acetal, and acetic acid were measured. Elemental analysis, performed on toxic, non-toxic elements, and oligoelements, 18 in total, revealed a wide variability of concentrations in both analytes and samples. High concentrations of $\mathrm{Cu}$ are sometimes evidenced, which are likely caused by losses from the distillation apparatus. The principal components analysis (PCA) allowed the differentiation of the ten volatile compounds quantified in two groups: the former, as described mainly by PC1, constituted by acetic acid, ethyl acetate, dietyl acetal, and acetaldehyde, and the second, described by PC2, constituted by 1-propanol, 2-methyl-1-propanol, the two coeluiting isomers 2-methyl-1-butanol and 3-methyl-1-butanol,1-hexanol, 2-phenylethanol, and 2,3-butanediol. Data obtained may be useful in order to establish a regulation for the production of high-quality traditional Filuferru from Sardinia.
\end{abstract}

Keywords: Filuferru; spirit; distillation; grape marc; volatile compounds; copper; trace elements; GC-MS; ICP-MS; PCA

\section{Introduction}

Traditional Filuferru is an ancient, high alcoholic strength beverage from Sardinia, Italy. For centuries, it is well-known and consumed by indigenous people not only as a beverage, but also as an ingredient in their traditional cuisine. Its artisanal manufacture took place in clandestine distilleries placed in domestic cellars or in the immediate neighbors of the vineyards, where usually wine (in the western side of Sardinia) or fermented grape marc (in the central or eastern side of the Isle) 
were distilled. Since unauthorized distillation was prohibited by law, the distillers buried the bottles of the product in order to occult them from inspections that were made by policemen, and only a wire (i.e., Filuferru in the Sardinian language) tied at the neck of the bottle and emerging from the soil level for few centimeters indicated the point where the valuable spirit was left. Craft distillation of Filuferru is still today accomplished by using an internally tinned copper pot still with a volume of usually between 25 and $100 \mathrm{dm}^{3}$. Figure 1 represents a typical apparatus for traditional batch distillation.

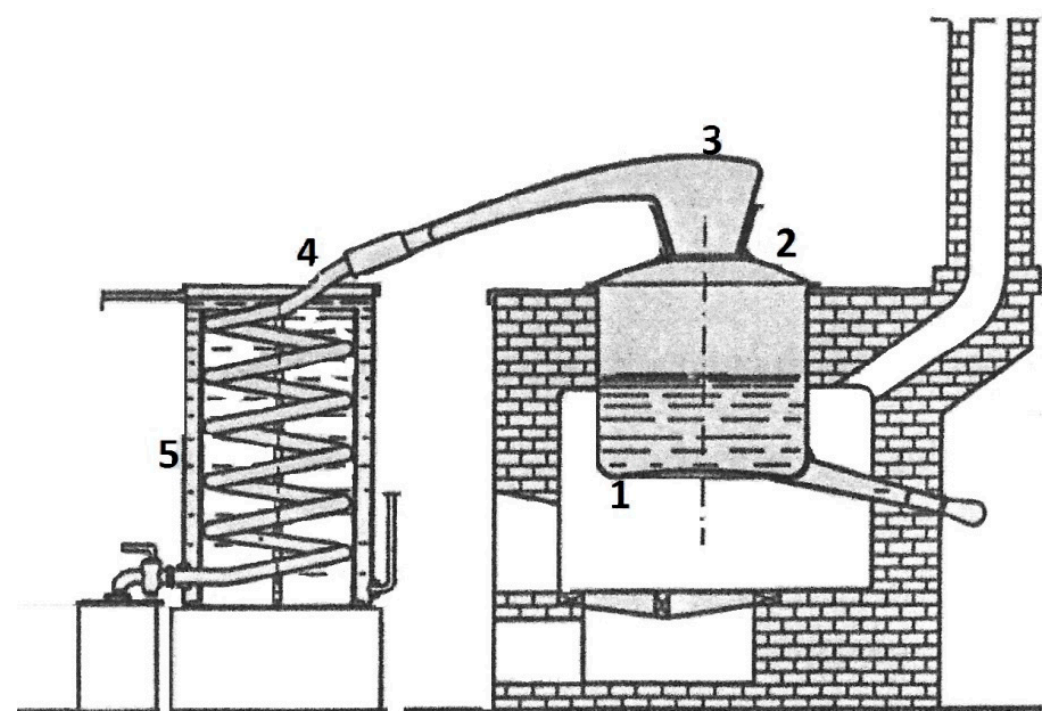

Figure 1. A typical pot still used for batch distillation of spirits. 1: Tinned copper pot; 2: still head; 3: swan's neck; 4: copper coil; and, 5: water cooling tank.

Heating of pot was accomplished by the combustion of wood or (preferably) liquid petroleum gas. Wine (or a mixture of fermented grape marc and water) is first fed in the pot and then heated, and great care was kept in order to avoid overheating of the charge. The temperature of the vapors measured on the still head is representative of their chemical composition, and this information is used by the distillers in order to separate the heart of the distillate from the head and the tail, both containing toxic and unpleasant substances, like methanol or high-boiling organic compounds, respectively. The heart of the distillate was hence condensed, giving rise to the final product, normally obtained in a single-stage distillation step. Traditional Filuferru is usually a colorless spirit, whose aromas recalled those of the wines (or the grape marc) of origin. Variants to the pure distillate are those aged or flavored with typical spontaneous essences of Sardinia, like strawberry tree (Arbutus Unedo L.) fruits, seeds of wild fennel, or hot pepper. Unlike Italian grappa (i.e., the distilled spirit by grape marc technologically most close to Filuferru) [1-6], to the best of our knowledge, no scientific publications concerning the chemical characterization of the main and the trace composition of traditional Filuferru are until now published, so the principal aim of the present contribution is to perform the qualitative and the quantitative determination of both principal and trace constituents of this ancient beverage. Hence, on a reliable sampling of Filuferru spirits distilled by grape marc collected by a number of craft producers from the Central Sardinia, the alcoholic strength, the chromatographic qualitative profile, the quantitative determination of ten of the principal volatile compounds beyond ethanol, and the amount of 18 trace elements (i.e., $\mathrm{Al}, \mathrm{B}, \mathrm{Ca}, \mathrm{Cd}, \mathrm{Cr}, \mathrm{Cu}, \mathrm{Fe}, \mathrm{K}, \mathrm{Mg}, \mathrm{Mn}, \mathrm{Na}, \mathrm{Ni}, \mathrm{Pb}, \mathrm{Sb}, \mathrm{Sn}, \mathrm{Sr}, \mathrm{W}$, and $\mathrm{Zn}$ ) have been measured and discussed. 


\section{Materials and Methods}

\subsection{Samples}

21 Filuferru samples produced from craft distillation of grape marc in eleven towns of central Sardinia (Figure 2) have been considered in this study.
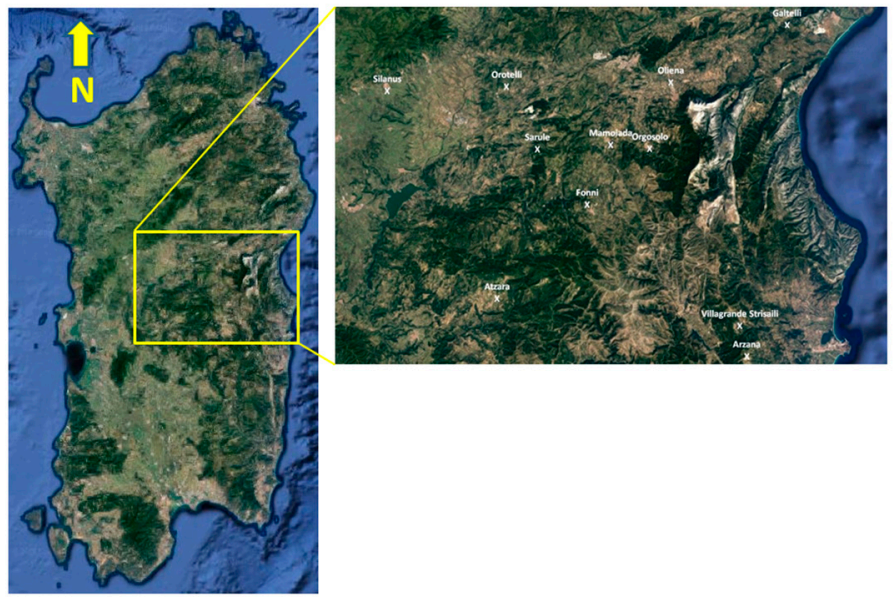

Figure 2. Geographical localization of the eleven towns of Central Sardinia where craft Filuferru samples considered in this study were produced.

A further commercial sample of Filuferru (sample 22) has been taken into account, just for comparisons sake. The geographical origin of craft samples of Filuferru is reported in Table 1. Beyond differences in the geographic origin of many samples, the choice of a small number of them was planned to put in evidence both analogies and differences ascribable to the nature of the grape marc or to the vintage effect. As a matter of fact, samples 18 and 19, from Orotelli, were produced by the same distiller while using grape marc from the same vineyard, but coming from two consecutive vintages, whereas samples 20 and 21, from Oliena, were produced in the same year by the same distiller using grape marc from two different vineyards. All of the samples were stored in glass bottles hermetically closed kept in the dark at $+4{ }^{\circ} \mathrm{C}$. Before each determination, samples were allowed to reach the room temperature.

Table 1. Geographical origin of craft samples of Filuferru (1-21) that were considered in the study.

\begin{tabular}{cc}
\hline Geographical Origin & Filuferru Sample \\
\hline Galtellì & 1 \\
Atzara & 2 \\
Orgosolo & 3 \\
Orotelli & $4^{\mathrm{a}}, 5^{\mathrm{a}}, 7^{\mathrm{a}}, 10^{\mathrm{a}}, 12,18^{\mathrm{b}}$ and $19^{\mathrm{b}}$ \\
Silanus & $6^{\mathrm{a}}$ and $9^{\mathrm{a}}$ \\
Sarule & $8^{\mathrm{a}}$ and $14^{\mathrm{a}}$ \\
Arzana & 11 \\
Villagrande Strisaili & 13 \\
Fonni & $15^{\mathrm{a}}$ and $17^{\mathrm{a}}$ \\
Mamoiada & 16 \\
Oliena & $20^{\mathrm{c}}$ and $21^{\mathrm{c}}$
\end{tabular}

a same town of production, different producer, different vineyard; ${ }^{b}$ same town of production, same producer, same vineyard, different year of production (two consecutive vintage); ${ }^{c}$ same town of production, same producer, same year of producion, different vineyard. 


\subsection{Chemicals and Reagents}

Ultrapure (Type 1) water (specific resistance $\geq 18 \mathrm{M} \Omega$ ) was used throughout the analyses. All reagents were at least of analytical grade. 100\% ethanol was purchased from Carlo Erba (Milan, Italy). Chromatographic standards (acetaldehyde, ethyl acetate, acetal, 1-propanol, 2-methyl-1-propanol, 2-methyl-1-butanol, 1-hexanol, acetic acid, 2,3-butanediol, and 2-phenylethanol) were from Sigma Aldrich (Milan, Italy). The elemental standard solutions were by: Carlo Erba (Milan, Italy) for $\mathrm{B}, \mathrm{K}, \mathrm{Mg}$ and $\mathrm{Na}\left(1000 \mathrm{mg} \mathrm{dm}^{-3}\right.$ in $2 \%(w / w)$ aqueous $\left.\mathrm{HNO}_{3}\right)$ and for $\mathrm{Cu}, \mathrm{Mn}, \mathrm{Pb}, \mathrm{Cr}, \mathrm{Zn}$, $\mathrm{Cd}$, and $\mathrm{Ni}\left(100 \mathrm{mg} \mathrm{dm}^{-3}\right.$ in $2 \%$ aqueous $\left.\mathrm{HNO}_{3}\right)$; Fluka (Milan, Italy) for $\mathrm{Al}$ and $\mathrm{Zn}\left(1000 \mathrm{mg} \mathrm{dm}^{-3}\right.$ in $2 \%$ aqueous $\mathrm{HNO}_{3}$ ); Sigma Aldrich (Milan, Italy) for $\mathrm{Ca}, \mathrm{W}$ and $\mathrm{Sb}\left(1000 \mathrm{mg} \mathrm{dm}^{-3}\right.$ in $2 \%$ aqueous $\mathrm{HNO}_{3}$ ) and VWR International (Milan, Italy) for Fe and $\mathrm{Sr}\left(1000 \mathrm{mg} \mathrm{dm}-3\right.$ in $2 \%$ aqueous $\left.\mathrm{HNO}_{3}\right)$. The $67 \%$ aqueous solution of $\mathrm{HNO}_{3}$ was an Ultrapure Normatom reagent (VWR International, Milan, Italy). The NexION ICP-MS tuning solution $\left(2 \% \mathrm{HNO}_{3}\right.$ solution in water containing $1 \mu \mathrm{g} \mathrm{dm}^{-3}$ each of $\mathrm{Be}, \mathrm{Ce}, \mathrm{Fe}, \mathrm{In}, \mathrm{Li}, \mathrm{Mg}, \mathrm{Pb}$, and $\mathrm{U}$, code N8145051) and the NexION ICP-MS KED tuning solution ( $1 \%$ HCI solution in water containing Co, $10 \mu \mathrm{g} \mathrm{dm}^{-3}$ and Ce, $1 \mu \mathrm{g} \mathrm{dm}^{-3}$, code N8145052) were both purchased from Perkin Elmer Italia (Monza, Italy).

\subsection{Instrumentation}

Ultrapure water was obtained by a New Human Power II Scholar UV apparatus (Human Corporation, Seoul, Korea). gas-chromatography-mass spectrometry (GC-MS) analysis was carried out using a gas-chromatograph model 7890, equipped with a VF-Wax $60 \mathrm{~m} \times 0.25 \mathrm{~mm}$ i.d., $0.5 \mu \mathrm{m}$ film thickness column, and coupled with a model 7000C MSD detector, all from Agilent Italy (Cernusco sul Naviglio, Italy). A Gerstel MPS autosampler and sample preparation robot Gerstel Italy (Cernusco sul Naviglio, Italy) was also coupled to the gas-chromatographic unit, whereas data were analyzed using a MassHunter Workstation B.06.00 SP1 (Agilent Italy (Cernusco sul Naviglio, Italy) running under Windows 7 environment. Semiquantitative determination of methanol has been accomplished with a gas-chromatograph model 8310, equipped with a Carbowax $30 \mathrm{~m} \times 1.0 \mathrm{~mm}$ i.d., $1.0 \mu \mathrm{m}$ film thickness column, and a LC-100 Laboratory Computing Integrator (Perkin Elmer, Monza, Italy). Elemental determinations were performed using an inductively coupled plasma mass spectrometry (ICP-MS) spectrometer model NexION 300X equipped with an autosampler model S10 (Perkin Elmer, Monza, Italy), running under the Windows 7 operating system.

\subsection{Analytical Methods}

\subsubsection{Alcoholic Strength by Volume (ASV)}

Alcoholic strength was measured according the EC Commission Regulation 606/09 [7]. Each measurement has been performed twice (in physical duplicate). Repeatability was better than $2 \%$, whereas average trueness, as measured on six synthetic ethanol/water mixtures (i.e., $40,45,50,55,60$, and $65 \% v / v)$, is always better than $3 \%$.

\subsubsection{GC-MS Analysis}

The chromatographic separation was accomplished using the following temperature program: $40{ }^{\circ} \mathrm{C}$ hold for $4 \mathrm{~min}$, then increased to $150{ }^{\circ} \mathrm{C}$ at a rate of $5.0^{\circ} \mathrm{C} \mathrm{min}-1$, held for $3 \mathrm{~min}$, then increased to $240{ }^{\circ} \mathrm{C}$ at a rate of $10^{\circ} \mathrm{C} \mathrm{min}-1$, and finally held for $12 \mathrm{~min}$. Helium was used as the carrier gas at a constant flow of $1 \mathrm{~cm}^{3} \mathrm{~min}^{-1}$. The identification of the individual components was performed by comparison with the co-injected pure compounds and by matching the MS fragmentation patterns and retention indices with the built in libraries or literature data or commercial mass spectral libraries (NIST/EPA/NIH 2008; HP1607), both purchased from Agilent Technologies (Cernusco sul Naviglio, Italy). The retention indices were measured analyzing an hydrocarbon mixture of n-alkanes (between 
C9 and C22) under the same chromatographic conditions that were used for analyzing samples in order to calculate the retention indexes with the generalized equation by Van den Dool and Kartz [8]

$$
I_{x}=100\left[\left(t_{x}-t_{n}\right) /\left(t_{n+1}-t_{n}\right)+n\right]
$$

where $t$ is the retention time, $x$ is the analyte, $n$ is the number of atoms of carbon of alkane that elutes before the analyte, and $n+1$ is the number of atoms of carbon of the alkane that elutes after the analyte.

Quantification has been accomplished by means an external calibration on three levels of concentration. A stock solution of each standard was prepared by weighting it accurately into a $10 \mathrm{~cm}^{3}$ volumetric flask. The sample was first dissolved in ethanol/water mixture $(70 \% v / v)$ and later the volume made up to the mark with the same mixture. The resulting stock solution was diluted with the same ethanol/water solution in order to obtain three reference solutions at decreasing concentrations of all the external standards that were considered. For each compound the calibration curves were made by linear regression by plotting the peak area of external standard against their known concentrations. The mixture of isomers 2-methyl-1-butanol and 3-methyl-1-butanol, overlapped in the chromatograms, were quantified with a calibration curve of one isomer (i.e., the 3-methyl-1-butanol), and results are expressed in terms of 3-methyl-1-butanol equivalent. Ethyl acetate and diethyl acetal were quantified by a selected ion monitoring (SIM) method setting the quadrupole for filter the ions 88.0 and 103.0 for ethyl acetate and diethyl acetal, respectively. The MS detector has been disabled between RT of 10.3 and $13 \mathrm{~min}$, in correspondence of the elution of ethanol, and this has caused the impossibility to evaluate the peak of methanol $(R I=907)$, very close to the ethanol's one $(R I=933)$. Each analysis was performed in triplicate, and the results were expressed as $\mathrm{mg} \mathrm{dm}^{-3}$.

\subsubsection{GC-FID Semiquantitative Determination of Methanol}

The semiquantitative gas-chromatographic-flame ionization detector (GC-FID) determination of methanol in Filuferru samples has been performed using the relevant temperature program: $35^{\circ} \mathrm{C}$ hold for $5 \mathrm{~min}$, then increased to $70{ }^{\circ} \mathrm{C}$ at a rate of $3.0^{\circ} \mathrm{C} \mathrm{min}{ }^{-1}$, and later increased to $180{ }^{\circ} \mathrm{C}$ at a rate of $15^{\circ} \mathrm{C} \mathrm{min}{ }^{-1}$. Nitrogen was used as the carrier gas at a constant flow of $10 \mathrm{~cm}^{3} \mathrm{~min}^{-1}$. The temperatures of the injector and of the detector were $230^{\circ} \mathrm{C}$ and $300{ }^{\circ} \mathrm{C}$, respectively, whereas the volume of solution injected was $0.2 \mathrm{~mm}^{3}$. Quantification of methanol has been accomplished by means an external calibration on three levels of concentration.

\subsubsection{ICP-MS Analysis}

The optimized parameters used during the ICP-MS measurements of the elemental amounts of $\mathrm{Al}, \mathrm{B}, \mathrm{Ca}, \mathrm{Cd}, \mathrm{Cr}, \mathrm{Cu}, \mathrm{Fe}, \mathrm{K}, \mathrm{Mg}, \mathrm{Mn}, \mathrm{Na}, \mathrm{Ni}, \mathrm{Pb}, \mathrm{Sb}, \mathrm{Sn}, \mathrm{Sr}, \mathrm{W}$, and $\mathrm{Zn}$ are: radiofrequency generator power output: $1600 \mathrm{~W}$; argon flows: plasma, $17.993 \mathrm{dm}^{3} \mathrm{~min}^{-1}$; nebulizer; $0.991 \mathrm{dm}^{3} \mathrm{~min}^{-1}$, auxiliary $1.203 \mathrm{dm}^{3} \mathrm{~min}^{-1}$; helium flow: $3.50 \mathrm{~cm}^{3} \mathrm{~min}^{-1}$; optimization on masses of ${ }^{7} \mathrm{Li},{ }^{24} \mathrm{Mg}$, ${ }^{115} \mathrm{In}$ and ${ }^{238} \mathrm{U}$; data acquisition: dwell time of $50 \mathrm{~ms}$, acquisition time of $3 \mathrm{~s}$; time to change modes: $60 \mathrm{~s}$; analytical masses: ${ }^{27} \mathrm{Al},{ }^{11} \mathrm{~B},{ }^{43} \mathrm{Ca},{ }^{111} \mathrm{Cd},{ }^{52} \mathrm{Cr},{ }^{63} \mathrm{Cu},{ }^{57} \mathrm{Fe},{ }^{39} \mathrm{~K},{ }^{24} \mathrm{Mg},{ }^{55} \mathrm{Mn},{ }^{23} \mathrm{Na},{ }^{60} \mathrm{Ni},{ }^{208} \mathrm{~Pb},{ }^{121} \mathrm{Sb},{ }^{118} \mathrm{Sn}$, ${ }^{88} \mathrm{Sr},{ }^{184} \mathrm{~W}$ and ${ }^{66} \mathrm{Zn}$. Al, Cr, $\mathrm{K}$, and $\mathrm{Mg}$ were always measured while using the He-collision mode, whereas the remaining elements were analyzed in the normal mode. Before accurate quantification, the tentative amounts of each analyte in all of tbe samples were evaluated by a TotalQuant approach. Quantification was always performed using external calibration with a $1 \mathrm{mg} \mathrm{dm}{ }^{-3} \mathrm{Rh}$ solution as an internal standard. The external calibration line was obtained on three different concentration levels for each analyte, ranging between the relevant LoQ value and the highest concentration evaluated in the semiquantitative analysis of all samples. The multielemental solutions have been prepared in physical triplicate for each concentration level, and each solution is taken into account in the assessment of the external calibration line. These solutions were prepared daily for proper dilution of intermediate stock solutions containing $10 \mathrm{mg} \mathrm{dm}^{-3}$ of each analyte in a 98:2 (v:v) water-ethanol solution. The detection limits were evaluated on the bases of measurements performed on the samples and the blank solution 
(a 98:2 (v:v) water-ethanol solution), respectively. In order to check the reliability of the analytical data, each five samples, a multielemental standard was reprocessed, and data obtained between this and the previous standard measurement were discarded if the last data obtained exceeded-for each analyte-the interval of confidence of $\bar{x} \pm 3$ s. Prior ICP-MS analysis, each Filuferru sample was 1:50 diluted with ultrapure water, hence these solutions were filtered off in a $0.45 \mu \mathrm{m}$ pore size polypropylene filter. Each sample was analyzed three times, and each analytical datum is the average of three replicated ICP-MS measurements.

\subsection{Multivariate Analysis}

Principal Component Analysis (PCA) were performed using a R-based software developed by the Group of Chemometrics of the Division of Analytical Chemistry of the Italian Chemical Society, freely downloadable from the site gruppochemiometria.it.

\section{Results and Discussion}

\subsection{Validation}

\subsubsection{GC-MS Method}

Validation of the quantitative GC-MS procedure of analysis of the volatile compounds has been accomplished mainly in terms of the definition of the interval of linearity and the precision, as measured in terms of the repeatability. Linearity was evaluated for each analyte within the real experimental concentration range, usually spanning for at least two (usually three) orders of magnitude of concentration as a function of the different analytes considered. Typical correlation coefficients $\mathrm{R}^{2}$ ranged between 0.994 and 0.998 . The $\mathrm{CV}$ of five consecutive measurements that were performed on the same sample in the same analytical session has been calculated. Each precision measurement has been accomplished, for each analyte, in the real sample showing the experimental concentration close to the average value. Typical precision values ranged between 2.6 and $6.4 \%$. Only ethyl acetate provided higher uncertainty values than those (i.e., 30\%). It is however interesting to note that, in a different Filuferru sample (i.e., sample 7, $1482 \pm 7 \mathrm{mg} \mathrm{dm}^{-3}$ ) showing a concentration of this analyte not too far from the average value $\left(1021 \mathrm{mg} \mathrm{dm}^{-3}\right)$, the precision for the same analyte (measured on three replications) was less than 1\%. Table 2 report the validation data for GC-MS quantitative measurements.

Table 2. Linearity and precision data for the GC-MS determination of volatile compounds in Filuferru samples.

\begin{tabular}{cccc}
\hline Compound & Linearity & Precision $(\boldsymbol{n}=\mathbf{5})$ \\
\hline & Range $\mathbf{( \mathbf { m g ~ d m } ^ { - 3 } )}$ & $\mathbf{R}^{\mathbf{2}}$ & CV Repeatability (Sample) \\
\hline Acetaldehyde & $10-10,000$ & 0.997 & $3.0(16)$ \\
Dietyl acetal & $1-10,000$ & 0.994 & $5.3(4)$ \\
Ethyl acetate & $10-10,000$ & 0.995 & $30(7)$ \\
1-propanol $^{-}$ & $10-1000$ & 0.998 & $4.8(13)$ \\
2-methyl-1-propanol & $10-1000$ & 0.997 & $6.4(20)$ \\
2-methyl-1-butanol + 3-methyl-1-butanol $^{\text {a }}$ & $50-5000$ & 0.996 & $5.7(4)$ \\
1-hexanol $^{\text {2-phenylethanol }}$ & $1-1000$ & 0.995 & $4.0(9)$ \\
2,3-butanediol b $^{\text {b- }}$ & $1-250$ & 0.996 & $2.6(17)$ \\
Acetic acid & $5-500$ & 0.995 & $3.1(18)$ \\
\hline
\end{tabular}

${ }^{a}$ both isomers coelute and results are expressed as 3-methyl-1-butanol equivalents, ${ }^{\text {b }}$ expressed as the sum of all isomers identified.

\subsubsection{ICP-MS Method}

For each element considered, the validation of the method has taken into account the limit of detection (LoD), limit of quantification (LoQ), the linearity, the precision (expressed on a real Filuferru 
sample as both repeatability and intermediate precision), and the bias (measured by recovery tests of each analyte on a real sample). LoD was calculated according to the Upper Limit Approach (ULA1) approved by IUPAC [9], whereas LoQ values have been established as 3.3 times the LoD value. Linearity was usually checked on at least two orders of magnitude of concentration, spanning in an interval from the relevant LoQ to $900 \mathrm{\mu g} \mathrm{dm}^{-3}$ as a function of the different analytes considered. Typical correlation coefficients $\mathrm{R}^{2}$ ranged between 0.992 and 0.999 . Precision was evaluated in terms of both repeatability and intermediate precision, whereas the bias was measured by means of recovery tests. Precision and trueness measurements have been accomplished on Filuferru real samples (i.e., samples 13, 18 and 20), fortified for Cr measurements with known amounts of analyte. Precision was evaluated in terms of both repeatability and intermediate precision. The CV of five consecutive measurements performed (a) on the same sample in the same analytical session, for repeatability evaluation; and (b) on the same sample on different analytical sessions across three weeks, for intermediate precision measurements, have been calculated. The acceptability of all precision figures was successfully checked according Horwitz's theory [10]. Trueness was measured in duplicate by means of recovery tests. Whereas, $\mathrm{Ca}, \mathrm{Cr}, \mathrm{Mg}, \mathrm{Mn}$, and $\mathrm{Pb}$ provided recoveries that were not statistically different from $100 \%$ (criteria: $t$-test, $p=0.95)$, the bias in the recovery amounts substantiated by the remaining elements was largely within the limits posed by the Association of Official Agricultural Chemists (AOAC) [11] for the concentration ranges considered. Table 3 reports the validation figures for the parameters above considered.

\subsection{Alcoholic Strength by Volume (ASV)}

Table 4 reports the ASV of the samples that were considered. The average ethanol amount measured in all of the samples analyzed is $49.9 \pm 0.2 \%(v / v)$, being the range of the craft products between $41.0 \pm 0.2$ and $62.4 \pm 0.4 \%(v / v)$. The measurements that were obtained confirm the reputation of high-alcoholic spirit of craft Filuferru: 10 samples on 21 showed ASV higher than 50\%, and one of them (i.e., the sample 13, from Villagrande Strisaili) was characterized by an ethanol amount even higher than $60 \%(v / v)$. It is interesting to note that samples 18 and 19 , from the same producer, but related to the distillation of grape marc obtained by the same vineyard in two consecutive vintages, show only a low difference between the relevant ASV, whereas even smaller differences were measured by the ASV of Filuferru 20 and 21 (same producer, same vintage, different vineyards). Finally, the commercial sample 22, from an industrial distillery, shows one of the lowest ASV (i.e., $41.8 \pm 0.6 \%$ ) measured in this study (higher only of that of the sample 1, $41.0 \pm 0.2 \%$ ).

\subsection{Analysis of Volatile Organic Compounds}

\subsubsection{Qualitative Analysis}

Many tens of volatile organic compounds coming from contributions of different production steps likely characterize the Filuferru aroma. Firstly, a meaningful contribution is due to compounds derived from the grape(s) variety: usually, they are free compounds, easily transferred to the spirit during distillation. Another contribution originates from compounds, like alcohols, acetates, and ethyl (or methyl) esters, formed during fermentation by metabolism of yeasts and bacteria. Finally, the last contribution is from compounds, like acetals, formed as a consequence of high temperature and alcohol concentration during the distillation process. Table 5 reports the qualitative attribution of GC-MS peaks obtained in the total ion current (TIC) chromatogram (reported in Figure 3) for the samples analyzed. 
Table 3. Validation data for the ICP-MS determination of elements in Filuferru samples.

\begin{tabular}{|c|c|c|c|c|c|c|c|}
\hline \multirow[t]{2}{*}{ Element } & \multicolumn{2}{|c|}{ Sensitivity $(n=3)$} & \multicolumn{2}{|l|}{ Linearity } & \multicolumn{2}{|c|}{ Precision $(n=5)$} & \multirow{2}{*}{$\begin{array}{c}\text { Bias }(n=2) \\
\text { Recovery }(\% \pm S D)\end{array}$} \\
\hline & $\operatorname{LoD}\left(\mu \mathrm{g} \mathrm{dm}^{-3}\right)$ & $\operatorname{LoQ}\left(\mu \mathrm{g} \mathrm{dm}^{-3}\right)$ & Concentration Range $\left(\mu \mathrm{g} \mathrm{dm}^{-3}\right)$ & $\mathbf{R}^{2}$ & Repeatability (CV) & Intermediate Precision (CV) & \\
\hline $\mathrm{Al}^{\mathrm{a}}$ & 0.08 & 0.26 & $0.26-26$ & 0.999 & $4.2^{b}$ & $3.2^{b}$ & $107 \pm 6^{b}$ \\
\hline B & 0.004 & 0.013 & $0.013-1.3$ & 0.998 & $2.1^{b}$ & $4.0^{\mathrm{b}}$ & $112 \pm 4^{b}$ \\
\hline $\mathrm{Ca}$ & 0.60 & 2.0 & $2-200$ & 0.997 & $0.4^{\mathrm{c}}$ & $0.8^{c}$ & $98 \pm 4^{c}$ \\
\hline $\mathrm{Cd}$ & 0.002 & 0.006 & $0.006-6$ & 0.999 & $10^{c}$ & $15^{c}$ & $110 \pm 1^{c}$ \\
\hline $\mathrm{Cr}^{\mathrm{a}}$ & 0.04 & 0.14 & $0.14-14$ & 0.992 & $15^{\mathrm{d}}$ & $15^{\mathrm{d}}$ & $102 \pm 7^{\mathrm{d}}$ \\
\hline $\mathrm{Cu}$ & 0.05 & 0.16 & $0.16-300$ & 0.998 & $2.9^{b}$ & $8.2^{b}$ & $90 \pm 5^{b}$ \\
\hline $\mathrm{Fe}$ & 0.13 & 0.44 & $0.44-44$ & 0.996 & $0.3^{\mathrm{e}}$ & $0.7^{\mathrm{e}}$ & $93 \pm 4^{\mathrm{e}}$ \\
\hline $\mathrm{K}^{\mathrm{a}}$ & 0.002 & 0.007 & $0.007-900$ & 0.997 & $0.7^{\mathrm{c}}$ & $0.2^{\mathrm{c}}$ & $92 \pm 3^{c}$ \\
\hline $\mathrm{Mg}^{\mathrm{a}}$ & 0.021 & 0.069 & $0.07-70$ & 0.994 & $1.8^{\mathrm{b}}$ & $2.4^{\mathrm{b}}$ & $98 \pm 4^{b}$ \\
\hline $\mathrm{Mn}$ & 0.012 & 0.040 & $0.04-4$ & 0.998 & $3.2^{b}$ & $2.5^{b}$ & $100 \pm 4^{b}$ \\
\hline $\mathrm{Na}$ & 0.16 & 0.53 & $0.6-300$ & 0.997 & $5.3^{b}$ & $9.0^{\mathrm{b}}$ & $90 \pm 3^{b}$ \\
\hline $\mathrm{Ni}$ & 0.019 & 0.065 & $0.065-6.5$ & 0.997 & $30^{\mathrm{e}}$ & $35^{\mathrm{e}}$ & $95 \pm 3^{e}$ \\
\hline $\mathrm{Pb}$ & 0.002 & 0.008 & $0.008-80$ & 0.996 & $0.4^{b}$ & $21^{\mathrm{b}}$ & $98 \pm 1^{\mathrm{b}}$ \\
\hline $\mathrm{Sb}$ & 0.005 & 0.018 & $0.018-1.8$ & 0.996 & $33^{b}$ & $30^{\mathrm{b}}$ & $111 \pm 3^{b}$ \\
\hline Sn & 0.056 & 0.18 & $0.18-18$ & 0.997 & $13^{b}$ & $17^{\mathrm{b}}$ & $110 \pm 2^{b}$ \\
\hline $\mathrm{Sr}$ & 0.004 & 0.013 & $0.013-1.3$ & 0.998 & $6.7^{b}$ & $12^{b}$ & $96.8 \pm 0.3^{b}$ \\
\hline $\mathrm{W}$ & 0.008 & 0.025 & $0.025-2.5$ & 0.998 & $13^{b}$ & $20^{\mathrm{b}}$ & $86 \pm 4^{b}$ \\
\hline $\mathrm{Zn}$ & 0.019 & 0.062 & $0.062-62$ & 0.998 & $7.0^{\mathrm{c}}$ & $2.2^{c}$ & $93 \pm 6^{b}$ \\
\hline
\end{tabular}

Prior to ICP-MS determination all Filuferru samples were 1:50 diluted with ultrapure water, ${ }^{\text {a }}$ element measured in He-collision mode; ${ }^{\mathrm{b}}$ measured on sample $20 ;{ }^{\mathrm{c}}$ measured on sample 18 ;

${ }^{\mathrm{d}} \mathrm{CV}$ measured after spiking Filuferru sample of a known amount of analyte up to reaching the lowest limit of the relevant interval of calibration; ${ }^{\mathrm{e}}$ measured on sample 13. 
Table 4. Alcoholic strength by volume (ASV) $(\%(v / v) \pm \mathrm{SD} ; n=2)$ and GC-MS quantification (mg $\left.\mathrm{dm}^{-3} \pm \mathrm{SD} ; n=3\right)$ of the principal volatile compounds in Filuferru samples.

\begin{tabular}{|c|c|c|c|c|c|c|c|c|c|c|c|}
\hline Sample & ASV (\% Vol) & Acetaldehyde & $\begin{array}{l}\text { Acetaldehyde } \\
\text { Diethyl Acetal }\end{array}$ & Ethyl Acetate & 1-Propanol & 2-Methyl-1-Propanol & 3-Methyl-1-Butanol a & 1-Hexanol & 2-Phenylethanol & 2,3-Butanediol a & $\begin{array}{l}\text { Acetic } \\
\text { Acid }\end{array}$ \\
\hline 1 & $41.0 \pm 0.2$ & $900 \pm 100$ & $250 \pm 10$ & $410 \pm 20$ & $275 \pm 20$ & $160 \pm 50$ & $950 \pm 60$ & $32 \pm 2$ & $31 \pm 0$ & $80 \pm 40$ & $485 \pm 40$ \\
\hline 2 & $50.2 \pm 0.2$ & $1710 \pm 90$ & $890 \pm 30$ & $900 \pm 30$ & $112 \pm 7$ & $330 \pm 20$ & $1546 \pm 4$ & $38 \pm 1$ & $58 \pm 1$ & $65 \pm 35$ & $555 \pm 15$ \\
\hline 3 & $58.2 \pm 0.2$ & $52 \pm 0$ & $5 \pm 0$ & $14 \pm 5$ & $55 \pm 8$ & $220 \pm 20$ & $390 \pm 60$ & $21 \pm 2$ & $20 \pm 2$ & $35 \pm 9$ & $110 \pm 10$ \\
\hline 4 & $46.8 \pm 0.0$ & $3650 \pm 150$ & $1530 \pm 80$ & $1230 \pm 40$ & $175 \pm 0$ & $530 \pm 10$ & $1220 \pm 70$ & $38 \pm 1$ & $27 \pm 0$ & $40 \pm 1$ & $450 \pm 50$ \\
\hline 5 & $54.0 \pm 0.0$ & $8000 \pm 1000$ & $3900 \pm 900$ & $4000 \pm 100$ & $370 \pm 60$ & $430 \pm 20$ & $1950 \pm 300$ & $70 \pm 10$ & $50 \pm 8$ & $100 \pm 100$ & $2600 \pm 300$ \\
\hline 6 & $51.2 \pm 0.0$ & $150 \pm 10$ & $51 \pm 7$ & $150 \pm 20$ & $43 \pm 3$ & $380 \pm 20$ & $350 \pm 30$ & $11 \pm 0$ & $11 \pm 0$ & $26 \pm 1$ & $134 \pm 7$ \\
\hline 7 & $52.2 \pm 0.2$ & $2900 \pm 500$ & $1400 \pm 400$ & $2550 \pm 800$ & $350 \pm 80$ & $220 \pm 40$ & $1310 \pm 20$ & $115 \pm 10$ & $27 \pm 0$ & $150 \pm 100$ & $1482 \pm 7$ \\
\hline 8 & $46.2 \pm 0.2$ & $1600 \pm 400$ & $700 \pm 100$ & $1100 \pm 200$ & $160 \pm 30$ & $340 \pm 30$ & $1600 \pm 300$ & $28 \pm 5$ & $70 \pm 20$ & $110 \pm 40$ & $700 \pm 200$ \\
\hline 9 & $47.6 \pm 0.4$ & $2230 \pm 70$ & $670 \pm 20$ & $890 \pm 20$ & $350 \pm 30$ & $450 \pm 30$ & $1740 \pm 90$ & $75 \pm 3$ & $66 \pm 5$ & $200 \pm 100$ & $1000 \pm 200$ \\
\hline 10 & $47.6 \pm 0.4$ & $1500 \pm 80$ & $610 \pm 80$ & $530 \pm 70$ & $170 \pm 50$ & $410 \pm 40$ & $800 \pm 200$ & $70 \pm 10$ & $28 \pm 0$ & $50 \pm 20$ & $360 \pm 50$ \\
\hline 11 & $52.2 \pm 0.2$ & $2300 \pm 100$ & $980 \pm 90$ & $1500 \pm 100$ & $350 \pm 40$ & $300 \pm 100$ & $1500 \pm 150$ & $39 \pm 5$ & $31 \pm 3$ & $50 \pm 20$ & $1140 \pm 50$ \\
\hline 12 & $49.0 \pm 0.2$ & $1800 \pm 500$ & $650 \pm 100$ & $700 \pm 100$ & $160 \pm 20$ & $240 \pm 30$ & $650 \pm 100$ & $23 \pm 3$ & $24 \pm 5$ & $80 \pm 30$ & $400 \pm 100$ \\
\hline 13 & $62.4 \pm 0.4$ & $6300 \pm 200$ & $630 \pm 500$ & $1400 \pm 100$ & $210 \pm 10$ & $220 \pm 20$ & $1200 \pm 300$ & $42 \pm 4$ & $29 \pm 5$ & $220 \pm 10$ & $540 \pm 60$ \\
\hline 14 & $50.0 \pm 0.0$ & $1000 \pm 300$ & $500 \pm 100$ & $1050 \pm 200$ & $190 \pm 40$ & $400 \pm 40$ & $1650 \pm 350$ & $26 \pm 5$ & $42 \pm 9$ & $55 \pm 20$ & $500 \pm 200$ \\
\hline 15 & $46.2 \pm 0.2$ & $1050 \pm 100$ & $340 \pm 10$ & $1520 \pm 40$ & $136 \pm 8$ & $358 \pm 7$ & $1640 \pm 80$ & $54 \pm 3$ & $50 \pm 10$ & $90 \pm 10$ & $1200 \pm 200$ \\
\hline 16 & $46.6 \pm 0.2$ & $490 \pm 6$ & $182 \pm 9$ & $290 \pm 10$ & $145 \pm 20$ & $400 \pm 60$ & $2100 \pm 100$ & $27 \pm 2$ & $71 \pm 6$ & $60 \pm 10$ & $180 \pm 20$ \\
\hline 17 & $45.8 \pm 1.0$ & $510 \pm 20$ & $124 \pm 7$ & $1170 \pm 60$ & $215 \pm 20$ & $200 \pm 100$ & $900 \pm 1200$ & $50 \pm 4$ & $38 \pm 1$ & $113 \pm 8$ & $1180 \pm 50$ \\
\hline 18 & $59.0 \pm 0.2$ & $1970 \pm 60$ & $1900 \pm 300$ & $1700 \pm 300$ & $180 \pm 20$ & $400 \pm 100$ & $1500 \pm 200$ & $50 \pm 5$ & $65 \pm 4$ & $99 \pm 3$ & $885 \pm 30$ \\
\hline 19 & $55.2 \pm 0.0$ & $1500 \pm 300$ & $800 \pm 80$ & $470 \pm 40$ & $210 \pm 30$ & $170 \pm 50$ & $670 \pm 90$ & $54 \pm 7$ & $27 \pm 8$ & $85 \pm 30$ & $400 \pm 100$ \\
\hline 20 & $49.2 \pm 0.4$ & $1210 \pm 70$ & $3000 \pm 300$ & $9000 \pm 1000$ & $400 \pm 20$ & $310 \pm 20$ & $1010 \pm 70$ & $7 \pm 0$ & $35 \pm 2$ & $80 \pm 9$ & $2530 \pm 60$ \\
\hline 21 & $46.2 \pm 0.2$ & $190 \pm 7$ & $42 \pm 1$ & $122 \pm 2$ & $187 \pm 4$ & $160 \pm 50$ & $600 \pm 20$ & $590 \pm 60$ & $80 \pm 20$ & $250 \pm 50$ & $5300 \pm 800$ \\
\hline $22^{c}$ & $41.8 \pm 0.6$ & $240 \pm 20$ & $55 \pm 3$ & $57 \pm 4$ & $260 \pm 60$ & $230 \pm 60$ & $400 \pm 100$ & $57 \pm 1$ & $15 \pm 9$ & $255 \pm 9$ & $310 \pm 20$ \\
\hline Average $^{d}$ & $49.9 \pm 0.2$ & 1875 & 873 & 1398 & 214 & 312 & 1167 & 69 & 41 & 104 & 1020 \\
\hline Range & $41.0 \div 62.4$ & $52 \div 8000$ & $5 \div 6300$ & $14 \div 9000$ & $43 \div 400$ & $160 \div 530$ & $350 \div 2100$ & $7 \div 590$ & $11 \div 80$ & $26 \div 250$ & $110 \div 5300$ \\
\hline
\end{tabular}

$\mathrm{SD}=$ standard deviation; ${ }^{\mathrm{a}}$ sum of both 2-methyl-1-butanol and 3-methyl-1-butanol isomers, and quantified in terms of 3-methyl-1-butanol equivalent concentration; ${ }^{\mathrm{b}}$ sum of both isomers; ${ }^{\mathrm{c}}$ commercial Filuferru sample, here considered only for comparison purposes; ${ }^{\mathrm{d}}$ average values calculated before rounding made for harmonizing the number of significant digits of each analytical data according the relevant uncertainty. 
Table 5. GC-MS qualitative attribution of volatile compounds in Filuferru samples.

\begin{tabular}{|c|c|c|c|c|c|c|c|c|c|c|c|c|c|c|c|c|c|c|c|c|c|c|c|}
\hline \multirow[t]{2}{*}{ Classes/Compounds } & \multicolumn{23}{|c|}{ Sample } \\
\hline & 1 & 2 & 3 & 4 & 5 & 6 & 7 & 8 & 9 & 10 & 11 & 12 & 13 & 14 & 15 & 16 & 17 & 18 & 19 & 20 & 21 & $22^{a}$ & RI \\
\hline Aldehydes & & & & & & & & & & & & & & & & & & & & & & & \\
\hline Acetaldehyde & $\mathrm{x}$ & $\mathrm{x}$ & - & $\mathrm{x}$ & $\mathrm{x}$ & $\mathrm{x}$ & $\mathrm{x}$ & $\mathrm{x}$ & $\mathrm{x}$ & $\mathrm{x}$ & $\mathrm{x}$ & $\mathrm{x}$ & $\mathrm{x}$ & $\mathrm{x}$ & $\mathrm{x}$ & $\mathrm{x}$ & $\mathrm{x}$ & $\mathrm{x}$ & $\mathrm{x}$ & $\mathrm{x}$ & $\mathrm{x}$ & $\mathrm{x}$ & 710 \\
\hline 3-methylbutanal & $\mathrm{x}$ & $\mathrm{x}$ & - & $\mathrm{x}$ & - & $\mathrm{x}$ & $\mathrm{x}$ & $\mathrm{x}$ & $\mathrm{x}$ & $\mathrm{x}$ & $\mathrm{x}$ & $\mathrm{x}$ & - & - & $x$ & $\mathrm{x}$ & - & - & $\mathrm{x}$ & $\mathrm{x}$ & - & $\mathrm{x}$ & 930 \\
\hline Nonanal & - & - & - & - & $\mathrm{x}$ & - & - & - & - & - & - & - & - & - & - & - & - & - & - & & - & - & 1409 \\
\hline Furfural & $\mathrm{x}$ & $\mathrm{x}$ & $\mathrm{x}$ & - & $\mathrm{x}$ & $\mathrm{x}$ & $\mathrm{x}$ & $\mathrm{x}$ & $\mathrm{x}$ & $\mathrm{x}$ & $\mathrm{x}$ & $\mathrm{x}$ & $\mathrm{x}$ & $\mathrm{x}$ & $\mathrm{x}$ & $\mathrm{x}$ & $\mathrm{x}$ & $\mathrm{x}$ & $\mathrm{x}$ & $\mathrm{x}$ & $x$ & $\mathrm{x}$ & 1496 \\
\hline Benzaldehyde & $\mathrm{x}$ & $\mathrm{x}$ & $\mathrm{x}$ & $\mathrm{x}$ & $\mathrm{x}$ & $\mathrm{x}$ & $\mathrm{x}$ & - & $\mathrm{x}$ & $\mathrm{x}$ & $\mathrm{x}$ & $\mathrm{x}$ & $\mathrm{x}$ & $\mathrm{x}$ & $\mathrm{x}$ & $\mathrm{x}$ & - & $\mathrm{x}$ & - & $\mathrm{x}$ & $\mathrm{x}$ & $\mathrm{x}$ & 1567 \\
\hline Ketones and hydroxyketones & & & & & & & & & & & & & & & & & & & & & & & \\
\hline Acetoin & $\mathrm{x}$ & $\mathrm{x}$ & $\mathrm{x}$ & $\mathrm{x}$ & $\mathrm{x}$ & $\mathrm{x}$ & $\mathrm{x}$ & $\mathrm{x}$ & $\mathrm{x}$ & $\mathrm{x}$ & $\mathrm{x}$ & $\mathrm{x}$ & $\mathrm{x}$ & $\mathrm{x}$ & $\mathrm{x}$ & $\mathrm{x}$ & $\mathrm{x}$ & $\mathrm{x}$ & $\mathrm{x}$ & $\mathrm{x}$ & - & - & 1333 \\
\hline 1-hydroxy-2-propanone & $\mathrm{x}$ & - & $\mathrm{x}$ & $\mathrm{x}$ & $\mathrm{x}$ & $\mathrm{x}$ & $\mathrm{x}$ & - & $\mathrm{x}$ & $\mathrm{x}$ & $\mathrm{x}$ & - & - & $\mathrm{x}$ & $\mathrm{x}$ & $\mathrm{x}$ & $\mathrm{x}$ & - & $\mathrm{x}$ & $\mathrm{x}$ & $\mathrm{x}$ & $\mathrm{x}$ & 1343 \\
\hline 2-hydroxy-2-cyclopenten-1-one & $\mathrm{x}$ & - & - & - & $\mathrm{x}$ & - & $\mathrm{x}$ & - & $\mathrm{x}$ & - & - & - & - & - & - & - & - & - & - & - & $\mathrm{x}$ & $\mathrm{x}$ & 1808 \\
\hline $\begin{array}{l}\text { Pyranone } \\
\text { Acetals }\end{array}$ & $\mathrm{x}$ & - & - & - & $\mathrm{x}$ & - & $\mathrm{x}$ & - & - & - & - & - & - & - & - & - & - & - & - & - & $\mathrm{x}$ & $\mathrm{x}$ & 2339 \\
\hline Acetaldehyde, ethyl methyl acetal & $\mathrm{x}$ & $\mathrm{x}$ & - & $\mathrm{x}$ & $\mathrm{x}$ & - & $\mathrm{x}$ & $\mathrm{x}$ & $\mathrm{x}$ & $x$ & $x$ & $x$ & - & - & $x$ & $x$ & - & $\mathrm{x}$ & $\mathrm{x}$ & - & $x$ & $\mathrm{x}$ & 854 \\
\hline Acetaldehyde, diethyl acetal & $\mathrm{x}$ & $\mathrm{x}$ & $\mathrm{x}$ & $\mathrm{x}$ & $\mathrm{x}$ & $\mathrm{x}$ & $\mathrm{x}$ & $\mathrm{x}$ & $\mathrm{x}$ & $\mathrm{x}$ & $\mathrm{x}$ & $\mathrm{x}$ & $\mathrm{x}$ & $\mathrm{x}$ & $\mathrm{x}$ & $\mathrm{x}$ & $\mathrm{x}$ & $\mathrm{x}$ & $\mathrm{x}$ & $\mathrm{x}$ & $\mathrm{x}$ & $\mathrm{x}$ & 907 \\
\hline Isovaleraldehyde, diethyl acetal & $\mathrm{x}$ & $\mathrm{x}$ & - & $\mathrm{x}$ & $\mathrm{x}$ & $\mathrm{x}$ & $\mathrm{x}$ & $\mathrm{x}$ & $\mathrm{x}$ & $\mathrm{x}$ & $\mathrm{x}$ & $\mathrm{x}$ & $\mathrm{x}$ & - & $\mathrm{x}$ & $\mathrm{x}$ & - & $\mathrm{x}$ & $\mathrm{x}$ & $\mathrm{x}$ & - & $\mathrm{x}$ & 1085 \\
\hline Acetaldehyde, ethyl amyl acetal & - & - & - & $\mathrm{x}$ & $\mathrm{x}$ & - & - & - & - & - & $\mathrm{x}$ & - & - & - & - & - & - & - & - & $\mathrm{x}$ & - & - & 1102 \\
\hline Hexanal, diethyl acetal & $\mathrm{x}$ & $\mathrm{x}$ & - & $\mathrm{x}$ & $\mathrm{x}$ & $\mathrm{x}$ & $\mathrm{x}$ & $\mathrm{x}$ & $\mathrm{x}$ & $\mathrm{x}$ & $\mathrm{x}$ & $\mathrm{x}$ & $\mathrm{x}$ & - & $\mathrm{x}$ & $\mathrm{x}$ & - & - & $\mathrm{x}$ & $\mathrm{x}$ & - & - & 1220 \\
\hline $\begin{array}{c}\text { 1,1-diethoxy-2-propanone } \\
\text { Esters }\end{array}$ & - & - & - & $\mathrm{x}$ & $\mathrm{x}$ & - & - & - & $\mathrm{x}$ & - & - & - & $\mathrm{x}$ & - & - & - & - & $\mathrm{x}$ & $\mathrm{x}$ & - & - & - & 1298 \\
\hline Ethyl acetate & $\mathrm{x}$ & $\mathrm{x}$ & $\mathrm{x}$ & $\mathrm{x}$ & $\mathrm{x}$ & $\mathrm{x}$ & $\mathrm{x}$ & $\mathrm{x}$ & $\mathrm{x}$ & $\mathrm{x}$ & $\mathrm{x}$ & $\mathrm{x}$ & $\mathrm{x}$ & $\mathrm{x}$ & $x$ & $\mathrm{x}$ & $\mathrm{x}$ & $\mathrm{x}$ & $\mathrm{x}$ & $\mathrm{x}$ & $\mathrm{x}$ & $\mathrm{x}$ & 901 \\
\hline Ethyl butanoate & - & $\mathrm{x}$ & - & - & - & - & - & - & - & - & - & - & - & $\mathrm{x}$ & $\mathrm{x}$ & $\mathrm{x}$ & - & $\mathrm{x}$ & - & & - & $\mathrm{x}$ & 1055 \\
\hline 3-methyl-1-butanol acetate & - & - & - & $\mathrm{x}$ & $\mathrm{x}$ & $\mathrm{x}$ & $\mathrm{x}$ & - & $\mathrm{x}$ & $\mathrm{x}$ & $\mathrm{x}$ & $\mathrm{x}$ & - & $\mathrm{x}$ & $\mathrm{x}$ & $\mathrm{x}$ & $\mathrm{x}$ & - & - & $\mathrm{x}$ & - & $\mathrm{x}$ & 1131 \\
\hline Ethyl hexanoate & - & - & - & - & $\mathrm{x}$ & - & $\mathrm{x}$ & - & - & - & - & - & $\mathrm{x}$ & - & - & - & - & $\mathrm{x}$ & $\mathrm{x}$ & $\mathrm{x}$ & - & $\mathrm{x}$ & 1238 \\
\hline Ethyl lactate & - & $\mathrm{x}$ & $\mathrm{x}$ & $\mathrm{x}$ & - & $\mathrm{x}$ & $\mathrm{x}$ & $\mathrm{x}$ & $\mathrm{x}$ & $\mathrm{x}$ & - & $\mathrm{x}$ & - & $\mathrm{x}$ & - & $\mathrm{x}$ & $\mathrm{x}$ & - & - & $\mathrm{x}$ & $\mathrm{x}$ & - & 1370 \\
\hline Ethyl octanoate & $\mathrm{x}$ & $\mathrm{x}$ & $\mathrm{x}$ & $\mathrm{x}$ & $\mathrm{x}$ & $\mathrm{x}$ & $\mathrm{x}$ & $\mathrm{x}$ & $\mathrm{x}$ & $\mathrm{x}$ & $\mathrm{x}$ & $\mathrm{x}$ & $\mathrm{x}$ & $\mathrm{x}$ & $\mathrm{x}$ & $\mathrm{x}$ & $\mathrm{x}$ & $\mathrm{x}$ & $\mathrm{x}$ & $\mathrm{x}$ & - & - & 1438 \\
\hline Ethyl decanoate & $\mathrm{x}$ & $\mathrm{x}$ & - & $\mathrm{x}$ & $\mathrm{x}$ & $\mathrm{x}$ & $\mathrm{x}$ & $\mathrm{x}$ & $\mathrm{x}$ & $\mathrm{x}$ & $\mathrm{x}$ & $\mathrm{x}$ & $\mathrm{x}$ & $\mathrm{x}$ & $\mathrm{x}$ & $\mathrm{x}$ & $\mathrm{x}$ & $\mathrm{x}$ & $\mathrm{x}$ & $\mathrm{x}$ & - & $\mathrm{x}$ & 1649 \\
\hline $\begin{array}{l}\text { Diethyl butanedioate } \\
\text { Alcohols, enols and polyols }\end{array}$ & $\mathrm{x}$ & $\mathrm{x}$ & $\mathrm{x}$ & $\mathrm{x}$ & $\mathrm{x}$ & $\mathrm{x}$ & $\mathrm{x}$ & $\mathrm{x}$ & $\mathrm{x}$ & $\mathrm{x}$ & $\mathrm{x}$ & $\mathrm{x}$ & $\mathrm{x}$ & $\mathrm{x}$ & $\mathrm{x}$ & $\mathrm{x}$ & $\mathrm{x}$ & - & $\mathrm{x}$ & $\mathrm{x}$ & $\mathrm{x}$ & - & 1693 \\
\hline Methanol ${ }^{\text {b }}$ & $\mathrm{x}$ & $\mathrm{x}$ & $\mathrm{x}$ & $\mathrm{x}$ & $\mathrm{x}$ & $\mathrm{x}$ & $\mathrm{x}$ & $\mathrm{x}$ & $\mathrm{x}$ & $\mathrm{x}$ & $\mathrm{x}$ & $\mathrm{x}$ & $\mathrm{x}$ & $x$ & $\mathrm{x}$ & $x$ & $\mathrm{x}$ & $\mathrm{x}$ & $\mathrm{x}$ & $\mathrm{x}$ & $\mathrm{x}$ & $x$ & \\
\hline 2-butanol & & & & & & & & & & & $x$ & & & & & & $\mathrm{x}$ & & & $\mathrm{x}$ & & $\mathrm{x}$ & 1042 \\
\hline 1-propanol & $\mathrm{x}$ & $\mathrm{x}$ & $\mathrm{x}$ & $\mathrm{x}$ & $\mathrm{x}$ & $\mathrm{x}$ & $\mathrm{x}$ & $\mathrm{x}$ & $\mathrm{x}$ & $\mathrm{x}$ & $\mathrm{x}$ & $\mathrm{x}$ & $\mathrm{x}$ & $\mathrm{x}$ & $\mathrm{x}$ & $\mathrm{x}$ & $\mathrm{x}$ & $\mathrm{x}$ & $\mathrm{x}$ & $\mathrm{x}$ & $\mathrm{x}$ & $\mathrm{x}$ & 1062 \\
\hline 2-methyl-1-propanol & $\mathrm{x}$ & $\mathrm{x}$ & $\mathrm{x}$ & $\mathrm{x}$ & $\mathrm{x}$ & $\mathrm{x}$ & $\mathrm{x}$ & $\mathrm{x}$ & $\mathrm{x}$ & $\mathrm{x}$ & $\mathrm{x}$ & $\mathrm{x}$ & $\mathrm{x}$ & $\mathrm{x}$ & $\mathrm{x}$ & $\mathrm{x}$ & $\mathrm{x}$ & $\mathrm{x}$ & $\mathrm{x}$ & $\mathrm{x}$ & $\mathrm{x}$ & $\mathrm{x}$ & 1117 \\
\hline 1-butanol & $\mathrm{x}$ & $\mathrm{x}$ & - & $\mathrm{x}$ & $\mathrm{x}$ & $\mathrm{x}$ & $\mathrm{x}$ & - & - & $\mathrm{x}$ & $\mathrm{x}$ & - & $\mathrm{x}$ & $\mathrm{x}$ & $\mathrm{x}$ & $\mathrm{x}$ & - & $\mathrm{x}$ & $\mathrm{x}$ & $\mathrm{x}$ & - & $\mathrm{x}$ & 1174 \\
\hline Pent-1-en-3-ol & - & $\mathrm{x}$ & - & $\mathrm{x}$ & $\mathrm{x}$ & $\mathrm{x}$ & $\mathrm{x}$ & $\mathrm{x}$ & - & - & $\mathrm{x}$ & $\mathrm{x}$ & $\mathrm{x}$ & - & $\mathrm{x}$ & - & - & $\mathrm{x}$ & $\mathrm{x}$ & $\mathrm{x}$ & - & $\mathrm{x}$ & 1184 \\
\hline 2-methyl-1-butanol + 3-methyl-1-butanol & $\mathrm{x}$ & $\mathrm{x}$ & $\mathrm{x}$ & $\mathrm{x}$ & $\mathrm{x}$ & $\mathrm{x}$ & $\mathrm{x}$ & $\mathrm{x}$ & $\mathrm{x}$ & $\mathrm{x}$ & $\mathrm{x}$ & $\mathrm{x}$ & $\mathrm{x}$ & $\mathrm{x}$ & $\mathrm{x}$ & $\mathrm{x}$ & $\mathrm{x}$ & $\mathrm{x}$ & $\mathrm{x}$ & $\mathrm{x}$ & $\mathrm{x}$ & $\mathrm{x}$ & 1234 \\
\hline 1-pentanol & $\mathrm{x}$ & $\mathrm{x}$ & $\mathrm{x}$ & $\mathrm{x}$ & $\mathrm{x}$ & $\mathrm{x}$ & $\mathrm{x}$ & $\mathrm{x}$ & $\mathrm{x}$ & $\mathrm{x}$ & $\mathrm{x}$ & $\mathrm{x}$ & $\mathrm{x}$ & & $\mathrm{x}$ & & - & $\mathrm{x}$ & $\mathrm{x}$ & $\mathrm{x}$ & $\mathrm{x}$ & - & 1272 \\
\hline 3-methylbut-3-en-1-ol & - & $\mathrm{x}$ & - & - & - & - & - & - & - & - & $\mathrm{x}$ & - & - & $\mathrm{x}$ & $\mathrm{x}$ & $\mathrm{x}$ & - & - & - & - & - & - & 1277 \\
\hline 2-ethyl-1-butanol & - & - & - & - & - & - & $\mathrm{x}$ & - & - & - & $\mathrm{x}$ & - & - & - & - & - & - & - & $\mathrm{x}$ & - & - & - & 1323 \\
\hline (Z)-pent-2-en-1-ol & $\mathrm{x}$ & $\mathrm{x}$ & $\mathrm{x}$ & $\mathrm{x}$ & $\mathrm{x}$ & $\mathrm{x}$ & $\mathrm{x}$ & $\mathrm{x}$ & $\mathrm{x}$ & $\mathrm{x}$ & $\mathrm{x}$ & $\mathrm{x}$ & $\mathrm{x}$ & - & $\mathrm{x}$ & $\mathrm{x}$ & - & $\mathrm{x}$ & $\mathrm{x}$ & $\mathrm{x}$ & $\mathrm{x}$ & - & 1339 \\
\hline 1-hexanol & $\mathrm{x}$ & $\mathrm{x}$ & $\mathrm{x}$ & $\mathrm{x}$ & $\mathrm{x}$ & $\mathrm{x}$ & $\mathrm{x}$ & $\mathrm{x}$ & $\mathrm{x}$ & $\mathrm{x}$ & $\mathrm{x}$ & $\mathrm{x}$ & $\mathrm{x}$ & $\mathrm{x}$ & $\mathrm{x}$ & $\mathrm{x}$ & $\mathrm{x}$ & $\mathrm{x}$ & $\mathrm{x}$ & - & $\mathrm{x}$ & $\mathrm{x}$ & 1366 \\
\hline (Z)-hex-3-en-1-ol & - & - & - & - & - & $\mathrm{x}$ & $\mathrm{x}$ & - & - & $\mathrm{x}$ & $\mathrm{x}$ & - & - & - & - & - & - & - & - & - & - & - & 1379 \\
\hline 3-ethoxy-1-propanol & $\mathrm{x}$ & - & $\mathrm{x}$ & $\mathrm{x}$ & $\mathrm{x}$ & $\mathrm{x}$ & $\mathrm{x}$ & $\mathrm{x}$ & $\mathrm{x}$ & $x$ & $\mathrm{x}$ & $\mathrm{x}$ & $\mathrm{x}$ & $\mathrm{x}$ & $\mathrm{x}$ & $\mathrm{x}$ & $\mathrm{x}$ & $\mathrm{x}$ & $\mathrm{x}$ & $\mathrm{x}$ & $\mathrm{x}$ & $\mathrm{x}$ & 1401 \\
\hline
\end{tabular}


Table 5. Cont.

\begin{tabular}{|c|c|c|c|c|c|c|c|c|c|c|c|c|c|c|c|c|c|c|c|c|c|c|c|}
\hline \multirow[t]{2}{*}{ Classes/Compounds } & \multicolumn{23}{|c|}{ Sample } \\
\hline & 1 & 2 & 3 & 4 & 5 & 6 & 7 & 8 & 9 & 10 & 11 & 12 & 13 & 14 & 15 & 16 & 17 & 18 & 19 & 20 & 21 & $22^{a}$ & RI \\
\hline \multicolumn{24}{|l|}{ Alcohols, enols and polyols } \\
\hline (Z)-hex-2-en-1-ol & - & - & - & - & - & - & - & - & - & - & $\mathrm{x}$ & - & $\mathrm{x}$ & - & - & $\mathrm{x}$ & - & - & - & $\mathrm{x}$ & - & - & 1419 \\
\hline Oct-1-en-3-ol & - & $\mathrm{x}$ & $\mathrm{x}$ & - & $\mathrm{x}$ & - & $\mathrm{x}$ & $\mathrm{x}$ & $\mathrm{x}$ & - & $\mathrm{x}$ & $\mathrm{x}$ & $\mathrm{x}$ & - & $x$ & - & - & $x$ & $\mathrm{x}$ & $\mathrm{x}$ & - & - & 1455 \\
\hline 1-heptanol & $\mathrm{x}$ & $\mathrm{x}$ & $\mathrm{x}$ & $\mathrm{x}$ & $\mathrm{x}$ & $\mathrm{x}$ & $\mathrm{x}$ & $\mathrm{x}$ & $\mathrm{x}$ & $\mathrm{x}$ & $x$ & $\mathrm{x}$ & $\mathrm{x}$ & $\mathrm{x}$ & $x$ & $\mathrm{x}$ & $\mathrm{x}$ & $x$ & $\mathrm{x}$ & - & - & - & 1463 \\
\hline Hept-4-en-1-ol & - & $\mathrm{x}$ & $\mathrm{x}$ & $\mathrm{x}$ & $\mathrm{x}$ & - & $\mathrm{x}$ & - & $\mathrm{x}$ & - & - & $\mathrm{x}$ & - & - & $\mathrm{x}$ & - & - & $x$ & $x$ & - & - & - & 1516 \\
\hline 2,3-butanediol isomer I & $\mathrm{x}$ & $\mathrm{x}$ & $\mathrm{x}$ & $\mathrm{x}$ & $\mathrm{x}$ & $\mathrm{x}$ & $\mathrm{x}$ & $\mathrm{x}$ & $\mathrm{x}$ & $\mathrm{x}$ & $x$ & $\mathrm{x}$ & $\mathrm{x}$ & $\mathrm{x}$ & $\mathrm{x}$ & $\mathrm{x}$ & $\mathrm{x}$ & $x$ & $\mathrm{x}$ & $\mathrm{x}$ & - & - & 1559 \\
\hline 2,3-butanediol isomer II & $\mathrm{x}$ & $\mathrm{x}$ & $\mathrm{x}$ & $\mathrm{x}$ & $\mathrm{x}$ & $\mathrm{x}$ & $\mathrm{x}$ & $\mathrm{x}$ & $\mathrm{x}$ & $\mathrm{x}$ & $\mathrm{x}$ & $\mathrm{x}$ & $\mathrm{x}$ & $\mathrm{x}$ & $\mathrm{x}$ & $\mathrm{x}$ & $\mathrm{x}$ & $x$ & $\mathrm{x}$ & $\mathrm{x}$ & $x$ & - & 1591 \\
\hline Propylene glycol & - & - & $\mathrm{x}$ & - & - & - & $\mathrm{x}$ & $\mathrm{x}$ & $\mathrm{x}$ & $\mathrm{x}$ & - & $\mathrm{x}$ & - & - & - & - & - & - & $x$ & - & $\mathrm{x}$ & - & 1610 \\
\hline 1-nonanol & $x$ & $\mathrm{x}$ & $\mathrm{x}$ & $\mathrm{x}$ & $\mathrm{x}$ & $\mathrm{x}$ & $\mathrm{x}$ & $\mathrm{x}$ & $\mathrm{x}$ & $x$ & $x$ & $\mathrm{x}$ & $x$ & - & $x$ & $\mathrm{x}$ & $x$ & $x$ & $x$ & $\mathrm{x}$ & - & - & 1670 \\
\hline 2-furanmethanol & $\mathrm{x}$ & - & $\mathrm{x}$ & $\mathrm{x}$ & $\mathrm{x}$ & $\mathrm{x}$ & $\mathrm{x}$ & - & - & - & - & - & - & - & - & - & - & - & - & - & - & - & 1682 \\
\hline$\alpha$-terpineol & $\mathrm{x}$ & - & - & - & - & $\mathrm{x}$ & $\mathrm{x}$ & - & - & $\mathrm{x}$ & $\mathrm{x}$ & - & - & - & - & $\mathrm{x}$ & $\mathrm{x}$ & - & $\mathrm{x}$ & - & - & - & 1723 \\
\hline 5-methyl-2-furanmethanol & $\mathrm{x}$ & - & - & - & - & - & $\mathrm{x}$ & - & - & - & - & - & - & - & - & - & - & - & - & - & - & - & 1743 \\
\hline 1-decanol & $\mathrm{x}$ & - & - & $\mathrm{x}$ & - & $\mathrm{x}$ & $\mathrm{x}$ & - & $\mathrm{x}$ & $\mathrm{x}$ & $\mathrm{x}$ & $\mathrm{x}$ & $\mathrm{x}$ & $\mathrm{x}$ & - & $\mathrm{x}$ & $\mathrm{x}$ & $\mathrm{x}$ & $\mathrm{x}$ & $\mathrm{x}$ & - & - & 1771 \\
\hline Citronellol & - & - & - & - & - & $\mathrm{x}$ & - & - & - & $\mathrm{x}$ & $\mathrm{x}$ & - & - & $\mathrm{x}$ & - & $\mathrm{x}$ & $\mathrm{x}$ & - & - & - & - & - & 1777 \\
\hline Phenylmethanol & $\mathrm{x}$ & $\mathrm{x}$ & $\mathrm{x}$ & $\mathrm{x}$ & - & - & $\mathrm{x}$ & $\mathrm{x}$ & $\mathrm{x}$ & $\mathrm{x}$ & $\mathrm{x}$ & $\mathrm{x}$ & - & $\mathrm{x}$ & $\mathrm{x}$ & $\mathrm{x}$ & $\mathrm{x}$ & $x$ & $\mathrm{x}$ & $\mathrm{x}$ & $\mathrm{x}$ & - & 1914 \\
\hline $\begin{array}{l}\text { 2-phenylethanol } \\
\text { Carboxylic acids }\end{array}$ & $\mathrm{x}$ & $\mathrm{x}$ & $\mathrm{x}$ & $\mathrm{x}$ & $\mathrm{x}$ & $\mathrm{x}$ & $\mathrm{x}$ & $\mathrm{x}$ & $\mathrm{x}$ & $x$ & $\mathrm{x}$ & $\mathrm{x}$ & $\mathrm{x}$ & $\mathrm{x}$ & $\mathrm{x}$ & $\mathrm{x}$ & $\mathrm{x}$ & $x$ & $\mathrm{x}$ & $\mathrm{x}$ & $\mathrm{x}$ & $x$ & 1954 \\
\hline Acetic acid & $\mathrm{x}$ & $\mathrm{x}$ & $\mathrm{x}$ & $\mathrm{x}$ & $\mathrm{x}$ & $\mathrm{x}$ & $\mathrm{x}$ & $\mathrm{x}$ & $\mathrm{x}$ & $\mathrm{x}$ & $\mathrm{x}$ & $\mathrm{x}$ & $x$ & $\mathrm{x}$ & $\mathrm{x}$ & $\mathrm{x}$ & $\mathrm{x}$ & $\mathrm{x}$ & $\mathrm{x}$ & $\mathrm{x}$ & $\mathrm{x}$ & $\mathrm{x}$ & 1473 \\
\hline 2-methylpropanoic acid & - & $\mathrm{x}$ & $\mathrm{x}$ & $\mathrm{x}$ & - & $\mathrm{x}$ & - & $\mathrm{x}$ & $\mathrm{x}$ & $\mathrm{x}$ & $\mathrm{x}$ & - & - & $\mathrm{x}$ & $\mathrm{x}$ & $x$ & - & - & - & - & - & - & 1588 \\
\hline 3-methylbutanoic acid & - & $\mathrm{x}$ & $\mathrm{x}$ & - & - & $\mathrm{x}$ & - & - & - & $x$ & $\mathrm{x}$ & $\mathrm{x}$ & - & $\mathrm{x}$ & $x$ & $\mathrm{x}$ & - & $x$ & $\mathrm{x}$ & - & $\mathrm{x}$ & $\mathrm{x}$ & 1690 \\
\hline Hexanoic acid & $\mathrm{x}$ & - & $\mathrm{x}$ & $\mathrm{x}$ & $\mathrm{x}$ & $\hat{x}$ & - & $\mathrm{x}$ & $\mathrm{x}$ & $\hat{x}$ & $\hat{x}$ & - & - & $\hat{x}$ & $\hat{x}$ & - & $x$ & - & $\mathrm{x}$ & - & - & $\mathrm{x}$ & 1865 \\
\hline Octanoic acid & $\mathrm{x}$ & - & - & $\mathrm{x}$ & $\mathrm{x}$ & - & $\mathrm{x}$ & - & - & - & $\mathrm{x}$ & - & - & - & - & - & $\mathrm{x}$ & - & - & - & $\mathrm{x}$ & $\mathrm{x}$ & 2075 \\
\hline $\begin{array}{l}\text { Decanoic acid } \\
\text { Hydrocarbons }\end{array}$ & $\mathrm{x}$ & - & - & - & $\mathrm{x}$ & - & $\mathrm{x}$ & - & - & - & - & - & - & - & - & - & $\mathrm{x}$ & $\mathrm{x}$ & $\mathrm{x}$ & $\mathrm{x}$ & - & $\mathrm{x}$ & 2287 \\
\hline 2,4-dimethyl-1-heptene & - & $x$ & - & - & - & - & $x$ & - & - & $\mathrm{x}$ & $\mathrm{x}$ & - & - & - & - & $\mathrm{x}$ & - & $x$ & - & - & $\mathrm{x}$ & & 883 \\
\hline
\end{tabular}

Compounds ( $\mathrm{x}=$ qualitatively present, - = qualitatively not detectable) are listed for classes according to the increasing retention time in VF-WAX column. RI: retention index; ${ }^{\text {a }}$ sample 22 is a commercial Filuferru sample, here considered only for comparison purposes; ${ }^{\mathrm{b}}$ the qualitative presence of methanol has been ascertained by means of a GC-FID method. 


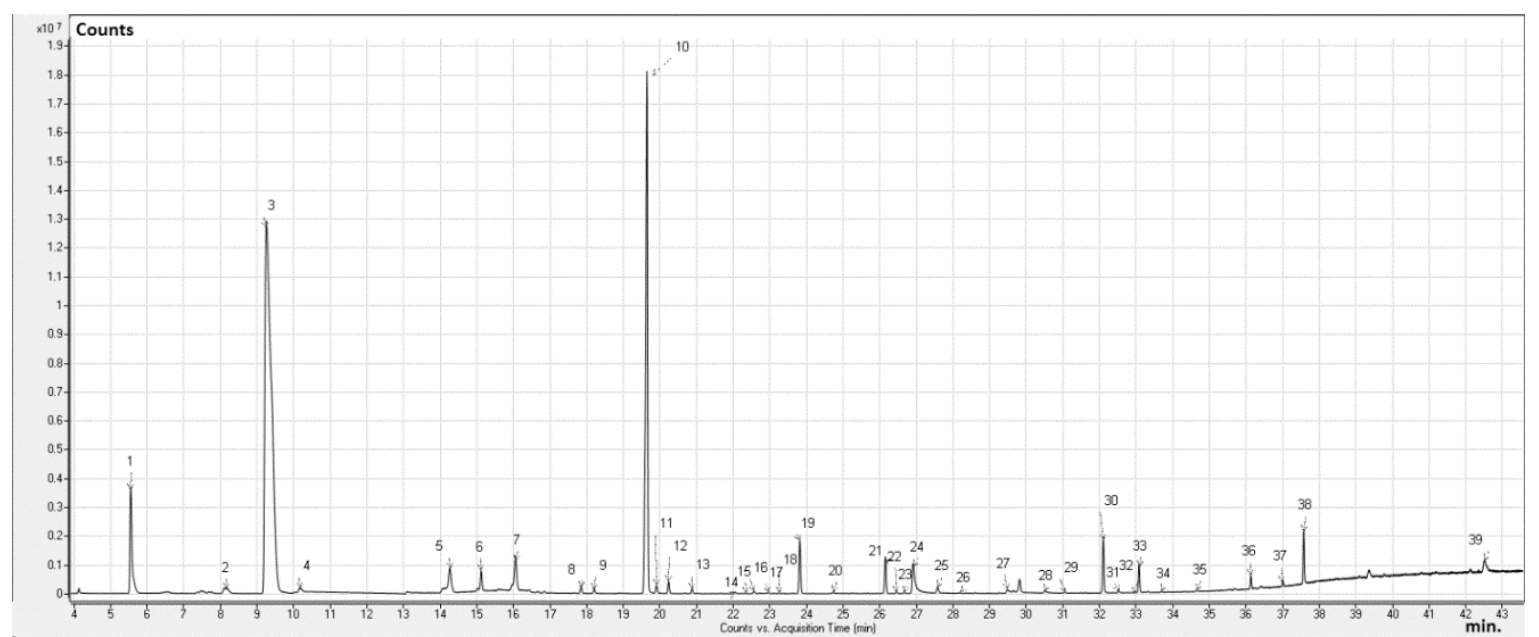

Figure 3. Typical GC-MS chromatogram of a Filuferru distillate by grape marc from Sardinia, Italy (sample 19, from Orotelli). Peak attribution: 1: Acetaldehyde; 2: Acetaldehyde, ethyl methyl acetal; 3: Ethyl acetate and acetaldehyde, diethyl acetal (not resolved); 4: 3-methyl-butanal; 5: 1-propanol; 6: Isovaleraldehyde, diethyl acetal; 7: 2-methyl-1-propanol; 8: 1-butanol; 9: Pent-1-en-3-ol; 10: 2-methyl-1-butanol and 3-methyl-1-butanol (not resolved); 11: Hexanal, diethyl acetal; 12: Ethyl hexanoate; 13: 1-pentanol; 14: 1,1-diethoxy-2-propanone; 15: 2-ethyl-1-butanol; 16: acetoin; 17: 2-(Z)-pent-2-en-1-ol; 18: 1-hydroxy-2-propanone; 19: 1-hexanol; 20: 3-ethoxy-1-propanol; 21: Ethyl octanoate; 22: Oct-1-en-3-ol; 23: 1-heptanol; 24: Acetic acid; 25: Furfural; 26: Hept-4-en-1-ol; 27: 2,3-butanediol (isomer I); 28: 2,3-butanediol (isomer II); 29: Propylene glycol; 30: Ethyl decanoate; 31: 1-nonanol; 32: 3-methylbutanoic acid; 33: Diethyl butanedioate; 34: $\alpha$-terpineol; 35: 1-decanol; 36: hexanoic acid; 37: Phenylmethanol; 38: 2-phenylethanol and, 39: n-decanoic acid.

60 compounds were identified, belonging to seven different chemical classes. In particular, five aldehydes, four among ketones and hydroxyketones, six acetals, eight esters and diesters, thirty among alcohols, enols and polyols, six carboxylic acids, and one linear alkene have been identified. Aldehydes and ketones are species normally formed during the alcoholic fermentation of grapes [12], and for this reason, they are present in the grape marc. Five of the six aldehydes are almost ubiquitous in the samples, whereas the nonanal was identified only in one (i.e., the sample 5) of the seven samples that were distilled in Orotelli. Among the ketones group, the acetoin and the 1-hydroxy-2-propanone were identified in almost all of the samples, whereas the 2-hydroxy-2-cyclopenten-1-one and the pyranone were contemporarily recognized only in samples $1,5,7,21$, and the commercial sample 22 (i.e., less than $25 \%$ of the total of the samples analyzed). Unlike aldehydes and ketones, acetals are a class of volatile compounds usually not present in the grape marc, but the reaction of acetalization occurs significantly during distillation due the concomitant effect of higher temperatures and concentration ethanol [13]. For these reasons, the acetaldehyde diethyl acetal (commonly termed "acetal") is always present in Filuferru samples. Only slightly less frequent is the qualitative identification in the samples of the acetaldehyde ethyl methyl acetal, of the hexanal diethyl acetal, and of the isovaleraldehyde diethyl acetal, all being recognized in more than $70 \%$ of the samples.

Whereas the presence of acetals is quite common also in grappa spirit, the presence of isovaleraldehyde diethyl acetal is not. This compound, recently observed in such matrix for the first time by Masino and coworkers [14], seems to be not so uncommon in the Filuferru composition. More rare is the identification of the acetaldehyde ethyl amyl acetal and of the 1,1-diethoxy-2-propanone, which are both present in not more than one-third of the samples. Among the different classes of volatile compounds, the esters are one of the most representative compounds in concurring to the definition of the aroma of the grape marc [15]. Among the lighter esters (up to five atoms of carbon), the ethyl acetate is always present, often as a principal constituent, in all Filuferru samples, whereas the 
ethyl octanoate, the ethyl decanoate, and the diethyl butanedioate are the esters at medium molecular weight (up to twelve atoms of carbon) that were identified in the almost totality of the samples. Also, the ethyl lactate (present in the grape marc due the malolactic fermentation) and the 3-methyl-1-butanol acetate have been frequently identified in more than the half of the samples, whereas more rare is the qualitative recognition of the ethyl butanoate and the ethyl hexanoate, which are present only in not more than one-third of the samples. It is not possible to ascertain in any sample of Filuferru the qualitative presence of esters of fatty acid, well-known to provide an unpleasant aroma to distilled spirits [15], whereas the presence of esters of low- and medium-molecular weight is responsible to attribute to Filuferru a fruity smell (light and undefined for ethyl acetate, more pronounced, and resembling the aroma of fruits, like apple, pear, banana, or pineapple for the medium molecular weight esters). The presence of ethyl esters (from ethyl acetate to ethyl decanoate) depends on fatty acid synthesis mediated by yeasts during the alcoholic fermentation [16].

As expected, alcohols are the class of compounds most represented in the qualitative analysis of volatile compounds in Filuferru. Obviously, beyond ethanol, practically all of the categories of alcohols from three to ten carbon atoms are represented in its volatile profile. Primary and secondary alcohols, with linear or branched carbon chain containing (or not) insaturations, are largely present in the composition of the beverage. Among others, 1-propanol, 2-methyl-1-propanol, the 2-phenylethanol, and the couple of isomers 2-methyl-1-butanol + 3-methyl-1-butanol (not completely resolved in the chromatographic separation) are always present in each sample examined. In addition, primary alcohols from $\mathrm{C} 4$ to $\mathrm{C} 10$, with the sole exception of 1-heptanol, are almost always present in more than the $90 \%$ of the volatile profile of Filuferru. Also, enols, like pent-1-en-3-ol, (Z)-pent-2-en-1-ol and oct-1-en-3-ol have been identified in more than 50\% of samples. Also present, albeit with a minor frequency, the unsubstituted and the 5-methyl substituted 2-furanmethanol, monoterpenoids from the grape like citronellol and $\alpha$-terpineol, and polyols as propylene glycol. Quite surprisingly, the 2-butanol has been retrieved only in very few Filuferru samples (i.e., the samples 11, 17, 20, and the commercial sample 22). This is unexpected, because it is well known in the literature that this alcohol concurs, together with the 1-propanol, the 3-methyl-1-butanol, and the 2-methyl-1-butanol (all present in meaningful amounts in the Filuferru spirit) to confer favorable key notes to distilled spirits [15]. In addition, 2-butanol is described in literature as a discriminant compound among wine and grape marc distillates, being basically absent in wine distillates, and constantly present in grape marc distillates [17]. Finally, acetic acid is obviously the most represented compound belonging to the group of the carboxylic acids. The recognizion of the 3-methylbutanoic acid has been frequent (in more than the half of the samples), whereas the qualitative detection of the 2-methylpropanoic acid, of the octanoic acid and of the decanoic acid (all present between the 35 and the 50\% of the samples analyzed) is less frequent. The GC-MS method that was previously described was unable to detect the presence of methanol, hence, in order to overcome this drawback, a semiquantitative GC-FID method has allowed for confirming the presence of the methanol in all of the samples considered.

\subsubsection{Quantitative Analysis}

Quantification has been accomplished on the most abundant and on the most frequently retrieved species that were identified in the previous qualitative step. Hence, Table 4 reports the concentrations of acetaldehyde, of dietyl acetal, of ethyl acetate, of acetic acid and of seven alcohols (i.e., 1-propanol, 2-methyl-1-propanol, 2-methyl-1-butanol, 3-methyl-1-butanol, 2-phenylethanol, and two isomers of the 2,3-butandiol). Very high concentrations of the lightest volatile compounds, like acetaldehyde, dietyl acetal, and ethyl acetate (average concentration of 1875,873 , and $1398 \mathrm{mg} \mathrm{dm}^{-3}$ ) are present in Filuferru spirits, whereas the total average alcoholic concentration (beyond ethanol) is $1907 \mathrm{mg} \mathrm{dm}^{-3}$. Finally, the average concentration of acetic acid is $1020 \mathrm{mg} \mathrm{dm}^{-3}$. For each analyte, the range spans over two (i.e., acetaldehyde, 1-hexanol, ethyl acetate, and acetic acid) but also three (i.e., diethyl acetate) orders of magnitude of concentration, also reaching amounts higher than $9 \mathrm{~g} \mathrm{dm}^{-3}$, as measured for ethyl acetate in sample 20. In the absence of any previous quantitative characterization of the volatile 
profile of the Filuferru, we tried a comparison between the data obtained in this study and the literature data from the same characterization made for the Italian Grappa, i.e., the distilled spirit that-for many aspects-is more close to Filuferru. Data reported in literature for the volatile composition of organic compounds in grappa are very often expressed in mass units on a volume of pure ethanol. Hence, for the sake of clarity and in order to facilitate the comparison of these results with the previous data, Table 6 reports the data contained in Table 4 converted in $\mathrm{mg} \mathrm{dm}^{-3}$ of pure ethanol.

By the comparison with the literature data for Grappa [14,18-23], it is evident that the craft Filuferru contains higher amounts of all compounds quantified in this study. Extending the comparison between the volatile composition of Filuferru and Grappa to similar European distillates from grape marc, like the Portuguese Bagaceira and the Spanish Orujo [18], the average amount of acetaldehyde found in craft Filuferru is really elevated (3640 $\mathrm{mg} \mathrm{dm}^{-3}$ of $100 \%$ ethanol), exceeding several times the maximum amount allowed in these Countries for the relevant spirits (i.e., $1000 \mathrm{mg} \mathrm{dm}^{-3} \mathrm{of}^{-}$ $100 \%$ ethanol). Also, the average concentration of ethyl acetate found in Filuferru (2767 $\mathrm{mg} \mathrm{dm}^{-3} \mathrm{of}^{-}$ $100 \%$ ethanol) exceeded the limit of $2500 \mathrm{mg} \mathrm{dm}^{-3}$ of $100 \%$ ethanol posed for this analyte. Before prematurely drawing any conclusion on the quality of the product, it is of the utmost importance to keep in mind that these values have been almost entirely measured on craft productions, accomplished in a traditional way and in the absence of any regulation of production. As a matter of fact, the amount of acetaldehyde and ethyl acetate in the commercial sample 22 (i.e., $570 \mathrm{mg} \mathrm{dm}^{-3}$ of $100 \%$ ethanol for acetaldehyde and $140 \mathrm{mg} \mathrm{dm}^{-3}$ of $100 \%$ ethanol for ethyl acetate) is well below not only the values measured for the craft productions (with the exception of sample 3 for both analytes and sample 6 for the acetaldehyde), but also the limits shared by Italy, Spain and Portugal for these compounds.

However, the high difference measured for both analytes among all craft Filuferru samples (two orders of magnitude) suggests a key influence of the technology of production (e.g., a wrong cut off of the heads in the distillation process as well as an incorrect fermentation process of the grape marc before distillation) on the concentration of acetaldehyde and ethyl acetate in the distillate. Furthermore, whereas high amounts of ethyl acetate in Filuferru can affect only sensorial properties, conferring to the beverage an intense and unpleasant herbaceous smell [15], a recent study [24] envisages potential health risk that is related to the consumption of alcoholic beverages containing high concentrations of acetaldehyde. Finally, the results from GC-FID semiquantitative determination of methanol of all samples of Filuferru show that this analyte is present only as a minor constituent of this distillate, with its concentration range between 30 and $150 \mathrm{mg} \mathrm{dm}^{-3}$ (the upper limit for Italian, Portuguese and Spanish distillates is fixed in $10000 \mathrm{mg} \mathrm{dm}^{-3}$ of $100 \%$ alcohol).

\subsection{Elemental Composition}

Table 7 reports the total concentration of 18 elements (i.e., $\mathrm{Al}, \mathrm{B}, \mathrm{Ca}, \mathrm{Cd}, \mathrm{Cr}, \mathrm{Cu}, \mathrm{Fe}, \mathrm{K}, \mathrm{Mg}, \mathrm{Mn}$, $\mathrm{Na}, \mathrm{Ni}, \mathrm{Pb}, \mathrm{Sb}, \mathrm{Sn}, \mathrm{Sr}, \mathrm{W}$, and $\mathrm{Zn}$ ) in 22 samples of Filuferru distilled spirit. Six elements of ascertained toxicity for humans, six oligoelements, and six elements generally recognized as not toxic have been chosen for this characterization. Data reported give account for a wide variability of concentration measured for almost all analytes. Whereas, the $\mathrm{Cr}$ concentration has always below the LoD for all samples analyzed, quantification for B has been possible-at levels of few parts for billion-only for samples 20 and 21, i.e., two Filuferru samples that were produced by the same distiller in the same vintage by grape marc from two different vineyards. 
Table 6. Amounts of the principal volatile compounds in Filuferru samples (data expressed as $\mathrm{mg} \mathrm{dm}^{-3}$ in ethanol 100\%).

\begin{tabular}{|c|c|c|c|c|c|c|c|c|c|c|}
\hline Sample & Acetaldehyde & $\begin{array}{l}\text { Acetaldehyde } \\
\text { Diethyl Acetal }\end{array}$ & $\begin{array}{c}\text { Ethyl } \\
\text { Acetate }\end{array}$ & 1-Propanol & 2-Methyl-1-Propanol & 3-Methyl-1-Butanol a & 1-Hexanol & 2-Phenylethanol & 2,3-Butanediol ${ }^{b}$ & Acetic Acid \\
\hline 1 & 2200 & 610 & 1000 & 670 & 400 & 2300 & 79 & 76 & 190 & 1200 \\
\hline 2 & 3400 & 1780 & 1800 & 220 & 660 & 3080 & 76 & 116 & 130 & 1100 \\
\hline 3 & 89 & 9 & 20 & 95 & 380 & 700 & 36 & 34 & 60 & 190 \\
\hline 4 & 7800 & 3300 & 2600 & 380 & 1130 & 2600 & 81 & 58 & 85 & 1000 \\
\hline 5 & 15,000 & 7000 & 7400 & 700 & 800 & 3600 & 130 & 90 & 200 & 4800 \\
\hline 6 & 300 & 100 & 300 & 85 & 740 & 690 & 21 & 21 & 51 & 26 \\
\hline 7 & 5500 & 2700 & 4900 & 700 & 420 & 2500 & 220 & 52 & 300 & 2900 \\
\hline 8 & 3500 & 1500 & 2400 & 350 & 735 & 3400 & 60 & 150 & 240 & 1500 \\
\hline 9 & 4700 & 1410 & 1900 & 730 & 945 & 3650 & 158 & 140 & 400 & 2100 \\
\hline 10 & 3100 & 1300 & 1110 & 360 & 860 & 1700 & 140 & 59 & 100 & 800 \\
\hline 11 & 4400 & 1900 & 2900 & 670 & 600 & 2800 & 76 & 60 & 100 & 2200 \\
\hline 12 & 4000 & 1300 & 1400 & 330 & 490 & 1300 & 47 & 50 & 160 & 800 \\
\hline 13 & 10,100 & 1010 & 2200 & 340 & 350 & 1900 & 67 & 46 & 350 & 900 \\
\hline 14 & 2000 & 1000 & 2100 & 380 & 800 & 3300 & 51 & 80 & 110 & 1000 \\
\hline 15 & 2300 & 730 & 3300 & 300 & 775 & 3600 & 120 & 120 & 190 & 2600 \\
\hline 16 & 1050 & 390 & 600 & 310 & 900 & 4500 & 58 & 150 & 130 & 390 \\
\hline 17 & 1120 & 270 & 2600 & 470 & 400 & 2000 & 110 & 82 & 250 & 2600 \\
\hline 18 & 3340 & 3200 & 2900 & 310 & 700 & 2550 & 85 & 111 & 168 & 1500 \\
\hline 19 & 2700 & 1450 & 850 & 380 & 310 & 1200 & 98 & 50 & 150 & 700 \\
\hline 20 & 2500 & 6100 & 18,200 & 800 & 630 & 2050 & 14 & 71 & 160 & 5100 \\
\hline 21 & 410 & 90 & 260 & 400 & 350 & 1300 & 1300 & 170 & 540 & 11000 \\
\hline $22^{c}$ & 570 & 130 & 140 & 600 & 550 & 960 & 136 & 40 & 600 & 740 \\
\hline Average ${ }^{d}$ & 3640 & 1694 & 2767 & 435 & 633 & 2349 & 144 & 83 & 212 & 2052 \\
\hline Range & $89 \div 15,000$ & $9 \div 7000$ & $20 \div 18,200$ & $85 \div 800$ & $310 \div 1130$ & $690 \div 4500$ & $14 \div 1300$ & $21 \div 170$ & $51 \div 600$ & $26 \div 11,000$ \\
\hline Range in Grappa e & $37 \div 1210$ & $27 \div 834$ & $37 \div 1744$ & $52 \div 2768$ & $222 \div 852$ & $364 \div 571$ & $3 \div 164$ & $1 \div 74$ & 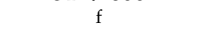 & $80 \div 97$ \\
\hline
\end{tabular}

a sum of both 2-methyl-1-butanol and 3-methyl-1-butanol isomers, and quantified in terms of 3-methyl-1-butanol equivalent concentration; ${ }^{\mathrm{b}}$ sum of both isomers; ${ }^{\mathrm{c}}$ commercial Filuferru sample, here considered only for comparison purposes; ${ }^{\mathrm{a}}$ average values calculated before rounding made for harmonizing the number of significant digits of each analytical data according the relevant uncertainty; ${ }^{\mathrm{e}}$ data from refs $[14,18-23] ;$ f analyte not previously quantified in this matrix. 
Table 7. Selected elemental abundances in Filuferru samples from Sardinia, Italy.

\begin{tabular}{|c|c|c|c|c|c|c|c|c|c|c|c|c|c|c|c|c|c|c|}
\hline Sample & $\mathrm{Al}$ & B & $\mathrm{Ca}$ & $\mathrm{Cd}$ & $\mathrm{Cr}$ & $\mathrm{Cu}$ & $\mathrm{Fe}$ & $\mathrm{K}$ & Mg & Mn & $\mathrm{Na}$ & $\mathrm{Ni}$ & $\mathrm{Pb}$ & sb & Sn & Sr & $\mathrm{w}$ & $\mathrm{Zn}$ \\
\hline 1 & $<13^{\mathrm{c}}$ & $<0.20^{d}$ & $<30^{\mathrm{d}}$ & $<0.32^{\mathrm{c}}$ & $<2.0^{\mathrm{d}}$ & $1483 \pm 1$ & $<6.7^{\mathrm{d}}$ & $<0.11^{\mathrm{d}}$ & $<1.0^{\mathrm{d}}$ & $8.2 \pm 0.3$ & $771 \pm 3$ & $37.5 \pm 0.7$ & $18.5 \pm 0.7$ & $3.1 \pm 0.1$ & $172 \pm 2$ & $13 \pm 3$ & $68 \pm 6$ & $148 \pm 1$ \\
\hline 2 & $<4.0^{\mathrm{d}}$ & $<0.20^{\mathrm{d}}$ & $<30^{\mathrm{d}}$ & $<0.32^{\mathrm{c}}$ & $<2.0^{\mathrm{d}}$ & $1096 \pm 4$ & $<6.7^{\mathrm{d}}$ & $108 \pm 6$ & $<1.0^{\mathrm{d}}$ & $<2.0^{\mathrm{c}}$ & $298 \pm 4$ & $6.1 \pm 0.1$ & $11.2 \pm 0.2$ & $1.4 \pm 0.2$ & $14.5 \pm 0.7$ & $6.2 \pm 0.2$ & $49.5 \pm 0.7$ & $92.9 \pm 0.1$ \\
\hline 3 & $94.5 \pm 0.5$ & $<0.20^{\mathrm{d}}$ & $251 \pm 1$ & $<0.32^{\mathrm{c}}$ & $<2.0^{\mathrm{d}}$ & $204 \pm 6$ & $299 \pm 1$ & $578 \pm 4$ & $80 \pm 1$ & $<2.0^{\mathrm{c}}$ & $390 \pm 20$ & $3.5 \pm 0.7$ & $1313 \pm 6$ & $<0.27^{\mathrm{d}}$ & $<2.8^{\mathrm{d}}$ & $10.5 \pm 0.7$ & $<0.38^{\mathrm{d}}$ & $43 \pm 3$ \\
\hline 4 & $19 \pm 1$ & $<0.20^{\mathrm{d}}$ & $118 \pm 2$ & $<0.32^{\mathrm{c}}$ & $<2.0^{\mathrm{d}}$ & $3996 \pm 6$ & $<6.7^{\mathrm{d}}$ & $760 \pm 20$ & $<1.0^{\mathrm{d}}$ & $3.4 \pm 0.5$ & $1720 \pm 15$ & $4.5 \pm 0.4$ & $183 \pm 1$ & $<0.27^{\mathrm{d}}$ & $12 \pm 6$ & $6.05 \pm 0.07$ & $23 \pm 3$ & $410 \pm 1$ \\
\hline 5 & $61 \pm 1$ & $<0.20^{\mathrm{d}}$ & $<30^{\mathrm{d}}$ & $<0.32^{\mathrm{c}}$ & $<2.0^{\mathrm{d}}$ & $77 \pm 4$ & $<6.7^{\mathrm{d}}$ & $<0.11^{\mathrm{d}}$ & $<1.0^{\mathrm{d}}$ & $2.2 \pm 0.3$ & $580 \pm 30$ & $<0.98^{\mathrm{d}}$ & $978 \pm 2$ & $<0.90^{\mathrm{c}}$ & $25 \pm 10$ & $4.5 \pm 0.7$ & $17.5 \pm 0.7$ & $<0.94^{\mathrm{d}}$ \\
\hline 6 & $<13^{\mathrm{c}}$ & $<0.20^{\mathrm{d}}$ & $125.5 \pm 0.7$ & $<0.32^{\mathrm{c}}$ & $<2.0^{\mathrm{d}}$ & $1450 \pm 100$ & $<6.7^{\mathrm{d}}$ & $2050 \pm 50$ & $<1.0^{\mathrm{d}}$ & $3.05 \pm 0.07$ & $1850 \pm 150$ & $<3.2^{\mathrm{c}}$ & $24 \pm 2$ & $<0.27^{\mathrm{d}}$ & $<9.2^{\mathrm{c}}$ & $4.6 \pm 0.6$ & $29 \pm 1$ & $25 \pm 6$ \\
\hline 7 & $98.3 \pm 0.9$ & $<0.20^{\mathrm{d}}$ & $770 \pm 50$ & $0.4 \pm 0.1$ & $<2.0^{\mathrm{d}}$ & $113 \pm 1$ & $<6.7^{\mathrm{d}}$ & $<0.11^{\mathrm{d}}$ & $145.5 \pm 0.5$ & $50.5 \pm 0.7$ & $710 \pm 10$ & $<0.98^{\mathrm{d}}$ & $171 \pm 8$ & $<0.27^{\mathrm{d}}$ & $16.5 \pm 0.7$ & $5.1 \pm 0.1$ & $13 \pm 1$ & $<0.94^{\mathrm{d}}$ \\
\hline 8 & $<13^{\mathrm{c}}$ & $<0.20^{\mathrm{d}}$ & $<30^{d}$ & $<0.32^{\mathrm{c}}$ & $<2.0^{\mathrm{d}}$ & $168.5 \pm 0.7$ & $<6.7^{\mathrm{d}}$ & $<0.11^{\mathrm{d}}$ & $<1.0^{\mathrm{d}}$ & $2.2 \pm 0.2$ & $1140 \pm 40$ & $<0.98^{\mathrm{d}}$ & $34 \pm 2$ & $<0.27^{\mathrm{d}}$ & $<2.8^{\mathrm{d}}$ & $4.4 \pm 0.5$ & $7.2 \pm 0.3$ & $<0.94^{\mathrm{d}}$ \\
\hline 9 & $45 \pm 1$ & $<0.20^{\mathrm{d}}$ & $204 \pm 1$ & $<0.32^{\mathrm{c}}$ & $<2.0^{\mathrm{d}}$ & $678 \pm 7$ & $<6.7^{\mathrm{d}}$ & $3000 \pm 60$ & $<1.0^{\mathrm{d}}$ & $6.1 \pm 0.1$ & $940 \pm 50$ & $5.4 \pm 0.6$ & $8.5 \pm 0.7$ & $<0.27^{\mathrm{d}}$ & $<9.2^{\mathrm{c}}$ & $2.8 \pm 0.3$ & $5.5 \pm 0.7$ & $688 \pm 7$ \\
\hline 10 & $<13^{\mathrm{c}}$ & $<0.20^{\mathrm{d}}$ & $<30^{\mathrm{d}}$ & $<0.32^{\mathrm{c}}$ & $<2.0^{\mathrm{d}}$ & $4000 \pm 50$ & $<6.7^{\mathrm{d}}$ & $<0.11^{\mathrm{d}}$ & $<1.0^{\mathrm{d}}$ & $<0.6^{\mathrm{d}}$ & $164 \pm 1$ & $<3.2^{\mathrm{c}}$ & $19 \pm 4$ & $<0.27^{\mathrm{d}}$ & $<9.2^{\mathrm{c}}$ & $2.5 \pm 0.7$ & $<0.38^{\mathrm{d}}$ & $56 \pm 4$ \\
\hline 11 & $24.05 \pm 0.07$ & $<0.20^{\mathrm{d}}$ & $1510 \pm 30$ & $<0.32^{\mathrm{c}}$ & $<2.0^{\mathrm{d}}$ & $780 \pm 40$ & $<6.7^{\mathrm{d}}$ & $630 \pm 10$ & $214.6 \pm 0.7$ & $<2.0^{\mathrm{c}}$ & $1700 \pm 600$ & $4.6 \pm 0.9$ & $128.5 \pm 0.7$ & $<0.27^{\mathrm{d}}$ & $<2.8^{\mathrm{d}}$ & $4.5 \pm 0.7$ & $<0.38^{\mathrm{d}}$ & $144 \pm 8$ \\
\hline 12 & $15.2 \pm 0.3$ & $<0.20^{\mathrm{d}}$ & $<98^{c}$ & $4.2 \pm 0.2$ & $<2.0^{\mathrm{d}}$ & $259 \pm 35$ & $<6.7^{\mathrm{d}}$ & $<0.11^{\mathrm{d}}$ & $<1.0^{d}$ & $2.4 \pm 0.2$ & $860 \pm 200$ & $<0.98^{\mathrm{d}}$ & $15 \pm 1$ & $<0.27^{\mathrm{d}}$ & $<2.8^{\mathrm{d}}$ & $5.5 \pm 0.8$ & $<0.38^{\mathrm{d}}$ & $48 \pm 1$ \\
\hline 13 & $<13^{c}$ & $<0.20^{\mathrm{d}}$ & $707 \pm 3$ & $<0.32^{\mathrm{c}}$ & $<2.0^{\mathrm{d}}$ & $180 \pm 1$ & $84 \pm 1$ & $250 \pm 20$ & $386 \pm 4$ & $<2.0^{c}$ & $2420 \pm 60$ & $4 \pm 1$ & $54 \pm 3$ & $<0.27^{\mathrm{d}}$ & $<9.2^{\mathrm{c}}$ & $4.2 \pm 0.2$ & $<0.38^{\mathrm{d}}$ & $112 \pm 4$ \\
\hline 14 & $<13^{\mathrm{c}}$ & $<0.20^{\mathrm{d}}$ & $<30^{\mathrm{d}}$ & $0.7 \pm 0.1$ & $<2.0^{\mathrm{d}}$ & $1730 \pm 25$ & $<6.7^{\mathrm{d}}$ & $21.5 \pm 0.7$ & $<1.0^{\mathrm{d}}$ & $<2.0^{\mathrm{c}}$ & $650 \pm 50$ & $45.3 \pm 0.4$ & $241 \pm 2$ & $<0.90^{\mathrm{c}}$ & $<2.8^{\mathrm{d}}$ & $2.4 \pm 0.6$ & $<0.38^{\mathrm{d}}$ & $930 \pm 10$ \\
\hline 15 & $<4.0^{\mathrm{d}}$ & $<0.20^{\mathrm{d}}$ & $<30^{\mathrm{d}}$ & $1.1 \pm 0.2$ & $<2.0^{\mathrm{d}}$ & $14,700 \pm 10$ & $<6.7^{\mathrm{d}}$ & $160 \pm 4$ & $<1$. & $3.5 \pm 0.7$ & $420 \pm$ & $4.4 \pm 0.6$ & $18.5 \pm 0.7$ & $<0.2$ & $<2.8^{\mathrm{d}}$ & $2.2 \pm 0.4$ & $8.6 \pm 0.6$ & $<0.94^{\mathrm{d}}$ \\
\hline 16 & $<13^{\mathrm{c}}$ & $<0.20^{\mathrm{d}}$ & $<30^{\mathrm{d}}$ & $<0.32^{\mathrm{c}}$ & $<2.0^{\mathrm{d}}$ & $86 \pm 4$ & $<6.7^{\mathrm{d}}$ & $6330 \pm 20$ & $<1$. & $<2.0$ & $1596 \pm 6$ & $<0.98^{\mathrm{d}}$ & $1.2=$ & $<0$. & $<2.8^{\mathrm{d}}$ & $1.95 \pm 0.07$ & $11=$ & $<0.94^{\mathrm{d}}$ \\
\hline 17 & $13.5 \pm 0.2$ & $<0.20^{\mathrm{d}}$ & $<30^{\mathrm{d}}$ & $<0.32^{\mathrm{c}}$ & $<2.0^{\mathrm{d}}$ & 17 & $<6.7^{\mathrm{d}}$ & $6550 \pm 10$ & $<1$. & $10.5 \pm 0.9$ & $804.6 \pm 0.5$ & $<0.98^{\mathrm{d}}$ & $6.9=$ & $<0.27^{\mathrm{d}}$ & $<9$ & $1.8 \pm 0.2$ & $3.8 \pm 0.2$ & $<0.94^{\mathrm{d}}$ \\
\hline 18 & $24.9 \pm 0.1$ & $<0.20^{\mathrm{d}}$ & $215.4 \pm 0.1$ & $0.5 \pm 0.3$ & $<2.0^{\mathrm{d}}$ & $210 \pm 10$ & $<6.7^{\mathrm{d}}$ & $1389 \pm 8$ & $<1.0^{\mathrm{d}}$ & $<2.0^{\mathrm{c}}$ & $970 \pm 30$ & $<3$ & $54 \pm 2$ & $2.2 \pm 0.3$ & $14=$ & $3.8 \pm 0.4$ & $<0.38^{\mathrm{d}}$ & $37 \pm 1$ \\
\hline 19 & $50 \pm 2$ & $<0.20^{\mathrm{d}}$ & $102 \pm 1$ & $<0.32^{\mathrm{c}}$ & $<2.0^{\mathrm{d}}$ & $860 \pm 20$ & $<6.7^{\mathrm{d}}$ & $387 \pm$ & $<1.0^{\mathrm{d}}$ & $2.1 \pm 0.2$ & $750=$ & $<3$. & 191.5 & $<0.2$ & $<2$. & $2.7 \pm$ & $<0.38^{\mathrm{d}}$ & $<0.94^{\mathrm{d}}$ \\
\hline 20 & $44 \pm 4$ & $1.94 \pm 0.08$ & $<30+2$ & $<0.32^{\mathrm{c}}$ & $<2.0^{\mathrm{d}}$ & $3480 \pm 20$ & $<6.7^{\mathrm{d}}$ & $40,900 \pm 200$ & $2800 \pm 200$ & $6.8 \pm 0.4$ & $2325 \pm 1$ & $<3$ & 16.5 & $18.9 \pm 0.2$ & $10.5 \pm 0.3$ & $1.5 \pm 0.7$ & $1.3 \pm 0.3$ & $<0.94^{\mathrm{d}}$ \\
\hline 21 & $20 \pm 8$ & $13.6 \pm 0.6$ & $<3$ & $<0.32^{\mathrm{c}}$ & $<2.0^{\mathrm{d}}$ & $470 \pm 70$ & $<6.7^{\mathrm{d}}$ & $3100 \pm 50$ & $<1.0$ & $<2.0^{c}$ & $432 \pm 2$ & $<3.2$ & $7.5 \pm$ & $<0.90^{\mathrm{c}}$ & $<2.8^{\mathrm{d}}$ & $3.8 \pm 0.3$ & $<1.25^{\mathrm{c}}$ & $<0.94^{\mathrm{d}}$ \\
\hline $22^{\mathrm{a}}$ & $20 \pm 2$ & $<0.20^{\mathrm{c}}$ & $<3$ & $<0.32^{\mathrm{c}}$ & $<2.0^{\mathrm{d}}$ & $<8$ & $<6.7^{\mathrm{d}}$ & $244 \pm 4$ & $<1.0$ & $<0.6^{\mathrm{d}}$ & $8600 \pm 40$ & $<0.98^{\mathrm{d}}$ & $<0$ & $<0$. & $<9.2^{\mathrm{c}}$ & $3.35 \pm 0.05$ & $19.5 \pm 0.7$ & $<0.94^{\mathrm{d}}$ \\
\hline Average b & $<24$ & $<0.89$ & $<203$ & $<0.43$ & $<2.0$ & $<1751$ & $<24$ & $<3022$ & $<166$ & 0.4 & 1368 & o. & $<159$ & $<1.53$ & $<15$ & 4.4 & $<12$ & $<125$ \\
\hline Range & $<4.0 \div 98.3$ & $<0.20 \div 13.6$ & $<30 \div 1510$ & $<0.10 \div 4.2$ & $<2.0$ & $<8 \div 14,700$ & $<6.7 \div 299$ & $<0.11 \div 40,900$ & $<1.0 \div 2800$ & $<2.0 \div 50.5$ & $164 \div 8600$ & $<0.98 \div 45.3$ & $<0.41 \div 1313$ & $<0.27 \div 18.9$ & $<2.8 \div 172$ & $1.5 \div 13$ & $<0.38 \div 68$ & $<0.94 \div 930$ \\
\hline
\end{tabular}

All elemental concentrations are expressed in $\mu \mathrm{g} \mathrm{dm}{ }^{-3}$; ${ }^{a}$ commercial Filuferru sample, distilled through a stainless steel apparatus rather than a copper apparatus, and here considered only for comparison purposes; balues preceded by a " $<$ " symbol are obtained considering in the calculation of the average value (calculated before rounding made for harmonizing the number of significant digits of each analytical data according the relevant uncertainty) at least one sample showing concentration below LoQ; ${ }^{\mathrm{c}}$ LoQ value; ${ }^{\mathrm{d}}$ LoD value. 
On the other hand, quantification of $\mathrm{Na}, \mathrm{Sr}, \mathrm{Cu}$, and $\mathrm{Pb}$ in craft Filuferru has been always possible, whereas the quantification of $\mathrm{Na}$ and $\mathrm{Sr}$ has been possible also for the commercial Filuferru sample, distilled in a stainless-steel apparatus rather than in a copper apparatus, and this-in our opinion-is the reason explaining the substantial absence of $\mathrm{Cu}$ by sample 22. The concomitant absence of $\mathrm{Pb}$ in sample 22 likely depends by the absence, in a stainless-steel apparatus, of any solders made using a $\mathrm{Sn} / \mathrm{Pb}$ filler material, typically that used for make solderings between parts in copper. Whereas, the concentration of $\mathrm{Na}$ is between 164 and $8600 \mu \mathrm{g} \mathrm{dm}^{-3}$, the amount of $\mathrm{Sr}$ is shifted downwards by two magnitude orders (i.e., between 1.5 and $15 \mu \mathrm{g} \mathrm{dm}{ }^{-3}$ ), while the range of $\mathrm{Cu}$ concentration is the widest among all of the elements considered (i.e., between the LoD, $8 \mu \mathrm{g} \mathrm{dm}{ }^{-3}$, and 14,700 $\mu \mathrm{g}$ $\left.\mathrm{dm}^{-3}\right)$. However, for all samples analyzed, the $\mathrm{Cu}$ amount is largely below the limit $\left(1000 \mathrm{mg} \mathrm{dm}^{-3}\right.$ of $100 \%$ ethanol) reported by Silva et al. [18]. The variability of the $\mathrm{Cu}$ and $\mathrm{Pb}$ concentrations in Filuferru is wide also within samples that were produced by the same distiller (i.e., Filuferru couples 18-19, produced in Orotelli in two consecutive vintage years, and 20-21, produced in Oliena in the same year by grape marc from two different vineyards). This variability should be tentatively ascribed by a number of different causes like: a different geochemical threshold for $\mathrm{Cu}$, the intensity of possible $\mathrm{Cu}$-based agrochemical treatments, the different bioaccumulation level of the elements on the grape marc as a function of the genotype or the pollution level and - not least-by the deterioration level of the copper pot still. In particular, the last one is the principal cause the contamination of spirits distilled in discontinuous copper apparatuses.

It is well known that the solubilization, by means of acidic alcoholic vapors, of compounds of $\mathrm{Cu}$ (II) like $\mathrm{CuCO}_{3} \cdot \mathrm{Cu}(\mathrm{OH})_{2}$ (the so-called "verdigris") $[25,26]$ formed on the inner copper surfaces of the copper pot, can contaminate the distillate. Even still EC and Italian legislations do not establish a maximum copper limit for distilled spirits, in Brazil the highest $\mathrm{Cu}$ concentration in alcoholic distillates is fixed in $5 \mathrm{mg} \mathrm{dm}^{-3}$ [27].

The highest concentrations of $\mathrm{Cu}$ and $\mathrm{Pb}$ measured in the Filuferru samples are $14,700 \mu \mathrm{g} \mathrm{dm}^{-3}$ for $\mathrm{Cu}$ (sample 15, produced in Fonni) and $1313 \mu \mathrm{g} \mathrm{dm} \mathrm{d}^{-3}$ for $\mathrm{Pb}$, (sample 3, produced in Orgosolo). $\mathrm{Sn}$ is the second element that is potentially correlated with the presence of solderings in a copper pot still. Unlike $\mathrm{Cu}$ and $\mathrm{Pb}, \mathrm{Sn}$ has been quantified only in less than one-third of the samples, with concentrations ranging between 10.5 and $172 \mu \mathrm{g} \mathrm{dm}^{-3}$. The concentration of another toxic element like $\mathrm{Cd}$ is rarely over the relevant LoQ: the element is quantified only on five samples, reaching the highest concentration in sample 12 ( $4.2 \mu \mathrm{g} \mathrm{dm}^{-3}$, from Orotelli). Oligoelements, like $\mathrm{Mn}, \mathrm{W}, \mathrm{Zn}$, and Ni have been quantified in more than the half of the samples $(\mathrm{Mn}, \mathrm{W}$, and $\mathrm{Zn})$, and in less than one third of them (Ni). The highest concentrations measured are on levels of few tens of $\mu \mathrm{g} \mathrm{dm}{ }^{-3}$ (for $\mathrm{Mn}, \mathrm{W}$, and $\mathrm{Ni}$ ) and almost a $\mathrm{mg} \mathrm{dm}^{-3}$ for $\mathrm{Zn}$. Potassium is the element showing the widest range (between LoD, $0.11 \mu \mathrm{g} \mathrm{dm}^{-3}$, and $40,900 \mu \mathrm{g} \mathrm{dm}^{-3}$ ). This is likely due also to the potential use of potassium-containing fertilizers in vine cultivation and/or the use of potassium metabisulfite as a source of sulfur dioxide in homemade winemaking processes. Metals, like Fe and Al, show a very different fate in Filuferru samples. Whereas $\mathrm{Al}$ is quite frequently quantified in them, reaching concentrations also close to $100 \mu \mathrm{g} \mathrm{dm}^{-3}$, Fe has been found only two times over the relevant LoD, and this happens to sample $3\left(299 \mu \mathrm{g} \mathrm{dm}^{-3}\right.$, from Orgosolo) and sample $13\left(84 \mu \mathrm{g} \mathrm{dm}{ }^{-3}\right.$, from Villagrande Strisaili). Although $\mathrm{Ca}$ is not commonly quantified in Filuferru (this happens only for eight samples), the frequency of quantification for $\mathrm{Mg}$ is also lower (only for five samples), and the concentrations measured for both analytes reached amounts of few thousands of $\mu \mathrm{g} \mathrm{dm}^{-3}$. Finally, Sb has been found over the relevant LoQ only in four samples, reaching its maximum amount on sample $20\left(18.9 \mu \mathrm{g} \mathrm{dm}^{-3}\right.$, from Oliena).

At the best of our knowledge, literature reports only very few contributions relating the determination of the trace elements in grape marc distillates, all aimed only on the evaluation of the $\mathrm{Cu}$ amount in similar distilled spirits from grape marc. Again, Grappa is chosen as the principal term of paragon for data obtained in this study. The amounts of $\mathrm{Cu}$ that were previously measured in this matrix ranged between 1.4 and $4.4 \mathrm{mg} \mathrm{dm}^{-3}$ (craft Grappa [25]) and from 0.4 to $0.7 \mathrm{mg} \mathrm{dm}^{-3}$ [28]. A wider comparison of our data, which keeps into account also some of other elements here measured, 
might be made with other traditional distilled spirits produced in other Countries. For example, very high $\mathrm{Cu}$ amounts (up to $105 \mathrm{mg} \mathrm{dm}^{-3}$ ) have been found in wine distillates from Crete, Greece [29], as well as in a spirituous beverage from Venezuela (Cocuy, that contains amounts of $\mathrm{Cu}$ between 4.5 and $86 \mathrm{mg} \mathrm{dm}^{-3}$ ) [30], whereas the amounts of $\mathrm{Cu}$ found in Zivania, a traditional Cypriot Spirit from distillation of grape marc [31], are comparable with data here reported for this element. Following the comparison between the trace amounts of elements in Filuferru and Zivania, it is possible to observe that the former prevails for the average concentrations of $\mathrm{Pb}$ and $\mathrm{Na}$, whereas the latter evidences higher amounts of $\mathrm{Ca}, \mathrm{Cr}, \mathrm{Mg}, \mathrm{Mn}$, and $\mathrm{Sb}$. Beyond $\mathrm{Cu}$, both of the distillates have comparable amounts of elements, like $\mathrm{Fe}, \mathrm{Al}$, and $\mathrm{Zn}$. On the other hand, the amounts of $\mathrm{Al}, \mathrm{Cd}, \mathrm{Mn}, \mathrm{Fe}, \mathrm{Cr}, \mathrm{Ni}, \mathrm{Pb}$, and $\mathrm{Zn}$ found in the wine distillates from Crete appear to be significantly higher than those found in Filuferru, as well as observed for the amounts of Fe and $\mathrm{Zn}$ measured in Cocuy distillate from agave. Also, the comparison between other literature data [32] substantiates that-at least for 14 elements of the 18 considered in this study-the average concentration and the range here reported does not differ significantly with respect to the amounts measured in previous studies.

\subsection{Principal Components Analysis.}

On first analysis, all 22 Filuferru samples, and all data from the quantitative analysis of organic volatile compounds (all analytes reported in Table 6, which have been provided data higher than the relevant LoQ for every sample) and all data from elements which concentration was over the relevant $\mathrm{LoQ}$ in at least $50 \%$ of samples (i.e., $\mathrm{Al}, \mathrm{Cu}, \mathrm{K}, \mathrm{Mn}, \mathrm{Na}, \mathrm{Pb}, \mathrm{Sr}$ and $\mathrm{Zn}$ ), for a total of 18 analytes, were kept into consideration for the Principal Component Analysis (PCA). Unfortunately, with this choice, the variance resulted very fragmented, as demonstrated by the amount of the total variance explained by the first two components, able to explain only less than $40 \%$ of the cumulative variance. Two PCA performed separately on the group of inorganic and volatile organic analytes, respectively, provided evidence that the highest amount of variance was explained by the last group; for this reason, all inorganic analytes were not more considered in the model. In this way, the cumulative variance explained by the two principal components rise up to $68 \%$ of the total variance. Hence, the $\mathrm{T}^{2}$ vs. Q diagnostic plot, as reported in Figure 4, provide evidence that sample 21 was clearly different from others, whereas samples 20 and-mainly-sample 13 were badly described, as revealed by its very high residual variance. For this reason, these data were considered outliers and removed from the dataset.

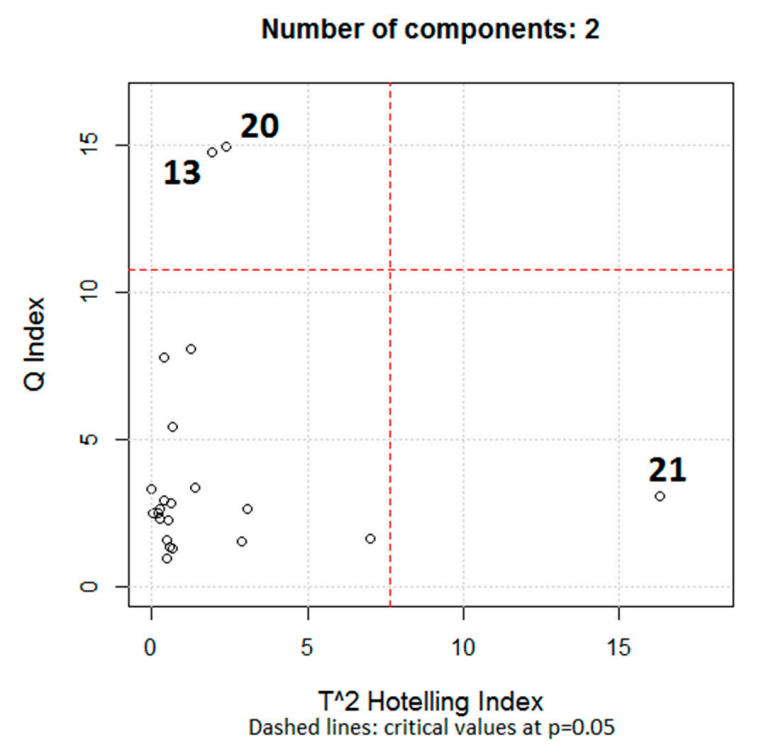

Figure 4. $\mathrm{T}^{2}$ vs. Q diagnostic plot of the Principal Component Analysis (PCA) performed on the whole Filuferru dataset. 
Hence, PCA analysis, which generates the biplot reported in Figure 5, referred to 19 scores and 10 objects.

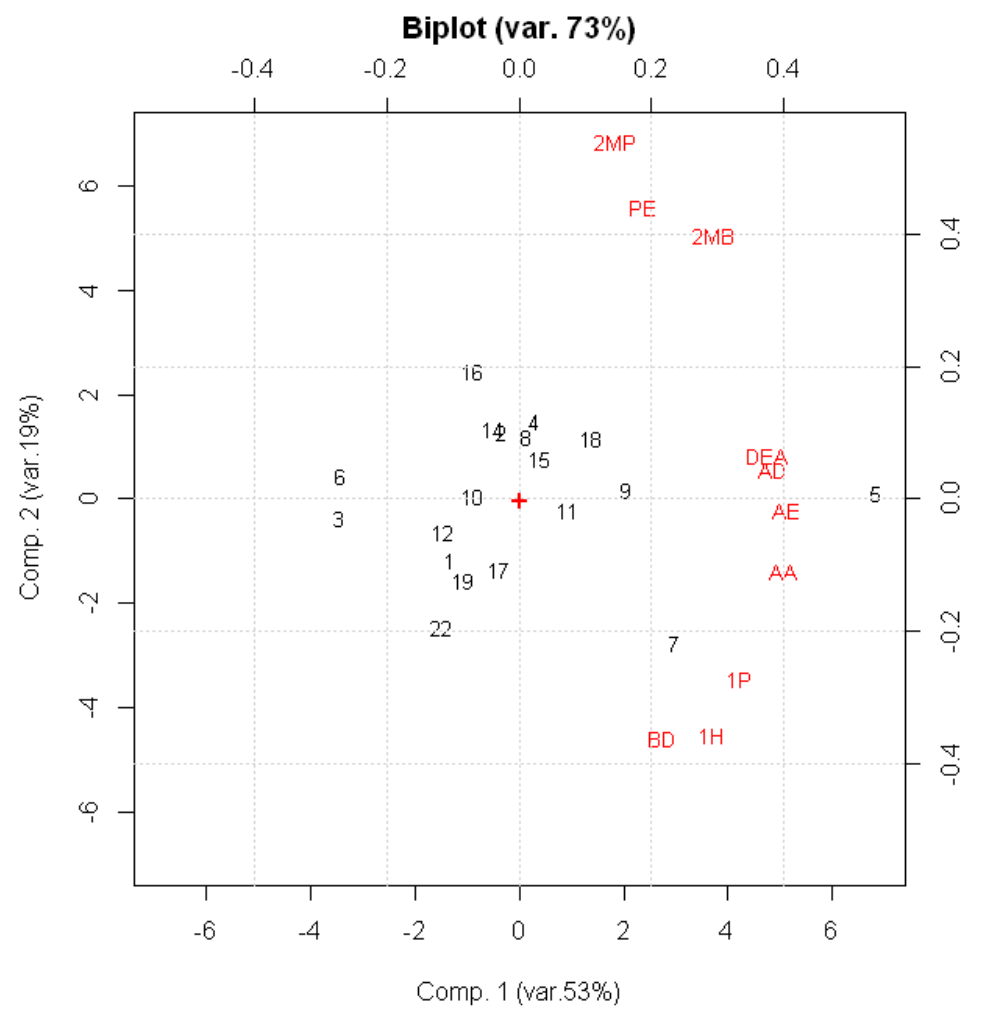

Figure 5. Biplot for PCA analysis (19 Filuferru samples, 10 analytical variables). Sample origin: 1, Galtellì; 2, Atzara; 3, Orgosolo; 4, 5, 7, 10, 12, 18 and 19, Orotelli; 6, 9, Silanus; 8, 14, Sarule; 11, Arzana; 15, 17, Fonni; 22, commercial Filuferru. Acronyms of volatile compounds; DEA, dietyl acetal; AD, acetaldehyde; AE, ethyl acetate; AA, acetic acid; 1P, 1-propanol; 2MP, 2-methyl-1-propanol; 2MB, the sum of 2-methyl-1-butanol and 3-methyl-1-butanol; 1H, 1-hexanol; PE, 2-phenylethanol; BD, 2,3-butanediol.

The two principal components were able to explain $73 \%$ of the total variance. From the biplot, it is evident that the dietyl acetal (DEA), the acetaldehyde (AD), the ethyl acetate (AE) and the acetic acid (AA) describe the PC1, whereas all of the alcohols (1-propanol, 1P; 2-methyl-1-propanol, 2MP; the two isomers 2-methyl-1-butanol and 3-methyl-1-butanol, both indicated as 2MB; 1-hexanol, $1 \mathrm{H}$; 2-phenylethanol, PE and the 2,3-butanediol, BD) describe the PC2. With the exception of sample 5, from Orotelli, and of samples 6 and 3 (from Silanus and Orgosolo, respectively), all of the remaining samples were described roughly in the same way by PC1 and PC2. In particular, sample 5 is characterized by high concentrations of low-boiling volatiles (i.e., DEA, AD, and $\mathrm{AE}$ ) and $\mathrm{AA}$, whereas samples 6 and 3 are different for a very low amounts of these compounds. PC2 differentiate samples 2, 4, 8, 14, 15, 16 and 18 by samples 1,12,17, 19 and 22 in terms of 2MB, 2MP, and PE (higher amounts for the first group rather than in the second group).

\section{Conclusions}

A preliminary screening of a wide number of craft samples of traditional Filuferru, a typical distillate of grape marc produced from centuries in Sardinia, Italy, has allowed, for the first time, to define a number of chemical properties like the alcoholic strength, the qualitative profile of the organic volatile compounds, the quantitative determination of the most abundant of them, and the quantitative determination of 18 trace elements. Data obtained have allowed to highlight a number of 
analogies and differences between the most close Italian spirit, the Grappa. The PC analysis allowed for the differentiation of the ten organic analytes being quantified in two groups: the first, described by PC1, accounting for acetic acid, ethyl acetate, dietyl acetal, and acetaldehyde, and the second, as described by PC2, accounted for the remaining six superior alcohols. The great variation in composition susbstantiates, on the one hand, in an incontrovertible way the artisanal nature of these spirits. On the other hand it reveals a number of potential quality issues (i.e., the highest concentration in the distillate of low-boiling point compounds and of acetic acid, and a quite high concentration of $\mathrm{Cu}$ ) that should keep into account in a next future in order to establish a regulation for this ancient and valuable traditional production. The key points where a careful quality control should be applied are the quality the grape marc, its fermentation and storage processes, the optimization of both the distillation phase, and the care and maintenance of the apparatus of distillation.

Author Contributions: Conceptualization, G.S., A.P., M.I.P., N.S.; Methodology, G.S., P.P.U.; Investigation, I.L., G.S., G.P., P.P.U.; Validation, I.L., G.S., G.P., P.P.U.; Chemometrics, M.C., G.P., P.P.U.; Data Curation, I.L., G.S., G.P., P.P.U.; Writing-Original Draft Preparation, I.L., G.S., G.P., P.P.U.; Writing-Review \& Editing, I.L., M.C., G.S., A.P., M.I.P., N.S., G.P., P.P.U.

Funding: This research received no external funding.

Acknowledgments: Authors gratefully thanks Giuseppina Pinna for the supply of the craft Filuferru samples.

Conflicts of Interest: The authors declare no conflict of interest.

\section{References}

1. Council Regulation (EEC) No 1576/89 of 29 May 1989 laying down general rules on the definition, description and presentation of spirit drinks. Off. J. Eur. Communities 1989, L160. Available online: https:/ / eur-lex. europa.eu/legal-content/EN/TXT/?uri=CELEX\%3A31989R1576 (accessed on 13 July 2018).

2. Matarese, L.; Festa, C.; Navarro, G. Caratteristiche chimiche della grappa, un distillato Italiano. Coefficienti di impurità. Riv. Vitic. Enol. 1969, 22, 255-285.

3. Usseglio-Tomasset, L. Studio chimico-analitico e gascromatografico della grappa piemontese. Vini d'Italia 1970, 12, 97-120.

4. Matarese, L.; Navarra, G. Le caratteristiche chimiche delle grappe venete-Nota II-Determinazione degli alcooli superiori per via gascromatografica. Riv. Vitic. Enol. 1971, 7, 257-261.

5. Versini, G.; Monetti, A.; Dalla Serra, A.; Inama, S. Analytical and statistical characterization of grappa from different Italian regions. In Les eaux de-vie Traditionnelles d'Origine Viticole; Bertrand, A., Ed.; Lavoisier-Tec \& Doc: Paris, France, 1991; pp. 137-150.

6. Da Porto, C. Grappa and Grape-Spirit Production. Crit. Rev. Biotechnol. 1998, 18, 13-24. [CrossRef]

7. Commission Regulation (EC) No 606/2009 of 10 July 2009 laying down certain detailed rules for implementing Council Regulation (EC) No 479/2008 as regards the categories of grapevine products, oenological practices and the applicable restrictions. Off. J. Eur. Communities 2009, L193. Available online: https: / / eur-lex.europa.eu/legal-content/en/ALL/?uri=CELEX:32009R0606 (accessed on 13 July 2018).

8. Van Den Dool, H.; Kratz, P.D. A generalization of the retention index system including linear temperature programmed gas-Liquid partition chromatography. J. Chromatogr. A 1963, 11, 463-471. [CrossRef]

9. Mocak, J.; Bond, A.M.; Mitchell, S.; Schollary, G. A statistical overview of standard (IUPAC and ACS) and new procedures for determining the limits of detection and quantification: Application to voltammetric and stripping techniques. Pure Appl. Chem. 1997, 69, 297-328. [CrossRef]

10. Horwitz, W. Evaluation of analytical methods used for regulation of foods and drugs. Anal. Chem. 1982, 54, 67A-76A. [CrossRef]

11. Association of Official Agricultural Chemists (AOAC) International. AOAC Peer Verified Methods Programs, Manual on Policies and Procedures; AOAC International: Arlington, VA, USA, 1998; Available online: http: / / citeseerx.ist.psu.edu/viewdoc/download?doi=10.1.1.196.7223\&rep=rep1\&type=pdf (accessed on 17 August 2018).

12. Ribéreau-Gayon, P.; Dubourdieu, D.; Donèche, B.; Lonvaud, A. Handbook of Enology, Volume 1: The Microbiology of Wine and Vinifications; John Wiley \& Sons Ltd.: Chichester, UK, 2006; ISBN 978-0-470-01034-1.

13. Misselhorn, K. Formation of acetals in rum: A kinetic study. Annal. Technol. Agric. 1975, 3-4, 371-381. 
14. Masino, F.; Montevecchi, G.; Riponi, C.; Antonelli, A. Composition of some commercial grappas (grape marc spirit): The anomalous presence of 1,1-diethoxy-3-methylbutane: A case study. Eur. Food Res. Technol. 2009, 228, 565-569. [CrossRef]

15. Versini, G.; Margheri, G. Rapporto fra i costituenti volatili della Grappa e le caratteristiche organolettiche. Vini d'Italia 1979, 122, 269-277.

16. Ribéreau-Gayon, P.; Glories, Y.; Manjean, A.; Dubourdieu, D. Handbook of Enology. Volume 2: The Chemistry of wine: Stabilization and Treatments; John Wiley \& Sons Ltd.: Chichester, UK, 2006; pp. 51-64.

17. Panighel, A. Studio dell'influenza delle differenti condizioni di stoccaggio delle vinacce sui composti aromatici varietali e di fermentazione. Tesi di Dottorato di Ricerca in viticoltura, enologia e marketing delle imprese vitivinicole. XXI Ciclo, Dipartimento di Biotecnologie Agrarie, Università degli Studi di Padova. 2009. Available online: http://paduaresearch.cab.unipd.it/1929/1/TESI_PanighelA.pdf (accessed on 13 July 2018).

18. Silva, M.L.; Macedo, A.C.; Malcata, F.X. Steam distilled spirits from fermented grape pomace. Food Sci. Technol. Int. 2000, 6, 295-300. [CrossRef]

19. Da Porto, C. Volatile composition of 'grappa low wines' using different methods and conditions of storage on an industrial scale. Int. J. Food Sci. Technol. 2002, 37, 395-402. [CrossRef]

20. Da Porto, C.; Decorti, D. Supercritical $\mathrm{CO}_{2}$ extraction of grappa volatile compounds. Int. J. Food Sci. Technol. 2009, 44, 1927-1932. [CrossRef]

21. Da Porto, C.; Natolino, A.; De Corti, D. Batch distillation of grappa: Effect of the recycling operation. Int. J. Food Sci. Technol. 2010, 45, 271-277. [CrossRef]

22. Cortés, S.; Rodríguez, R.; Salgado, J.M.; Domínguez, J.M. Comparative study between Italian and Spanish grape marc spirits in terms of major volatile compounds. Food Control 2011, 22, 673-680. [CrossRef]

23. Matias-Guiu, P.; Rodríguez-Bencomo, J.J.; Pérez-Correa, J.R.; López, F. Aroma profile design of wine spirits: Multi-objective optimization using response surface methodology. Food Chem. 2018, 245, 1087-1097. [CrossRef] [PubMed]

24. Paiano, V.; Bianchi, G.; Davoli, E.; Negri, E.; Fanelli, R.; Fattore, E. Risk assessment for the Italian population of acetaldehyde in alcoholic and non-alcoholic beverages. Food Chem. 2014, 154, 26-31. [CrossRef] [PubMed]

25. Baldo, M.A.; Daniele, S. Voltammetric monitoring and speciation of copper ions in Italian "Grappa" with platinum microelectrodes. Electroanalytical 2006, 18, 633-639. [CrossRef]

26. Alberti, G.; Guiso, M.G.; Biesuz, R. Usage of EmporeTM membrane in alcoholic media for copper(II) distribution studies. Talanta 2009, 79, 603-612. [CrossRef] [PubMed]

27. Complementation on Identification and Quality Standards for Alcoholic Distillates. Official Journal Brazil; Decree No. 371; Brasilian Ministry of Agriculture: Brasília, Brazil, 1974.

28. Tamasi, G.; Donati, A.; Leone, G.; Magnani, A.; Cini, R.; Macchia, E.; Rossi, C.; Bonech, C. Grappa quality from the Chianti and Montepulciano areas (Tuscany, Italy): Monitoring the leaching of copper from distillation columns. Int. J. Food Sci. Technol. 2018, 53, 1558-1565. [CrossRef]

29. Galani-Nikolakaki, S.; Kallithrakas-Kontos, N.; Katsanos, A.A. Trace element analysis of Cretan wines and wine products. Sci. Total Environ. 2002, 285, 155-163. [CrossRef]

30. Hernandez-Caraballo, E.A.; Avila-Gomez, R.M.; Capote, T.; Rivas, F.; Perez, A. Classification of Venezuelan spirituous beverages by means of discriminant analysis and artificial neural networks based on their $\mathrm{Zn}, \mathrm{Cu}$ and Fe concentrations. Talanta 2003, 60, 1259-1267. [CrossRef]

31. Kokkinofta, R.; Petrakis, P.V.; Mavromoustakos, T.; Theocharis, C.R. Authenticity of the traditional cypriot spirit "Zivania" on the basis of metal content using a combination of coupled plasma spectroscopy and statistical analysis. J. Agric. Food Chem. 2003, 51, 6233-6239. [CrossRef] [PubMed]

32. Szymczycha-Madeja, A.; Welna, M.; Jamroz, P.; Lesniewicz, A.; Pohl, P. Advances in assessing the elemental composition of distilled spirits using atomic spectrometry. Trends Anal. Chem. 2014, 64, 127-135. [CrossRef]

(C) 2018 by the authors. Licensee MDPI, Basel, Switzerland. This article is an open access article distributed under the terms and conditions of the Creative Commons Attribution (CC BY) license (http:/ / creativecommons.org/licenses/by/4.0/). 